(n)

trobertivier Journal of Nonlinear Mathematical Physics

\title{
Robin boundary condition and shock problem for the focusing nonlinear Schro̊dlinger equation
}

Spyridon Kamvissis, Dmitry Shepelsky, Lech Zielinski

To cite this article: Spyridon Kamvissis, Dmitry Shepelsky, Lech Zielinski (2015) Robin boundary condition and shock problem for the focusing nonlinear Schrödinger equation, Journal of Nonlinear Mathematical Physics 22:3, 448-473, DOI:

https://doi.org/10.1080/14029251.2015.1079428

To link to this article: https://doi.org/10.1080/14029251.2015.1079428

Published online: 04 January 2021 


\title{
Robin boundary condition and shock problem for the focusing nonlinear Schrödinger equation
}

\author{
Spyridon Kamvissis \\ Department of Applied Mathematics, University of Crete \\ Knossos-Heraklion, 71409, Crete, Greece \\ spyros@tem.uoc.gr \\ Dmitry Shepelsky \\ B. Verkin Institute for Low Temperature Physics and Engineering \\ 47 Lenin Avenue, 61103 Kharkiv, Ukraine \\ shepelsky@yahoo.com \\ Lech Zielinski \\ Laboratoire de Mathématiques Pures et Appliquées, \\ Université du Littoral Côte d'Opale \\ 50 rue Ferdinand Buisson - B.P. 69962228 Calais, France \\ Lech.Zielinski@lmpa.univ-littoral.fr
}

Received 13 February 2015

Accepted 19 June 2015

\begin{abstract}
We consider the initial boundary value (IBV) problem for the focusing nonlinear Schrödinger equation in the quarter plane $x>0, t>0$ in the case of periodic initial data, $u(x, 0)=\alpha \exp (-2 \mathrm{i} \beta x)$ (or asymptotically periodic, $u(x, 0)-\alpha \exp (-2 \mathrm{i} \beta x) \rightarrow 0$ as $x \rightarrow \infty)$, and a Robin boundary condition at $x=0: u_{x}(0, t)+q u(0, t)=0, q \neq 0$. Our approach is based on the unified transform (the Fokas method) combined with symmetry considerations for the corresponding Riemann-Hilbert $(\mathrm{RH})$ problems. We present the representation of the solution of the IBV problem in terms of the solution of an associated $\mathrm{RH}$ problem. This representation also allows us to determine an initial value (IV) problem, of a shock type, a solution of which being restricted to the half-line $x>0$ is the solution of the original IBV problem. In the case $\beta<0$, the large-time asymptotics of the solution of the IBV problem is presented in the "rarefaction" sector, demonstrating, in particular, an oscillatory behavior of the boundary values in the case $q>0$, contrary to the decay to 0 in the case $q<0$.
\end{abstract}

Keywords: initial boundary value problems; oscillatory initial data; nonlinear Schrödinger equation.

2010 Mathematics Subject Classification: 35Q55, 35Q15, 37K10, 37K40

\section{Introduction}

Any adaptation of the inverse scattering transform (IST) method to the study of initial boundary value (IBV) problems for nonlinear evolution equations possessing a Lax pair representation (called integrable) faces a major problem: the evolution of the spectral data require knowledge of an "excessive" amount of boundary values: they cannot all be given as boundary conditions for a well-posed IBV problem. However, certain particular classes of boundary conditions, under which the IBV problem remains completely integrable, i.e. solving it reduces to solving a series of well-posed linear problems, can be specified. 
In the case of decaying initial data, these classes (for various nonlinear equations) are known to be related to an appropriate continuation, based on the Bäcklund transformation, of the given initial data to the whole axis, which reduces the study of the IBV problem to the study of the associated initial value (IV) problem. Although this was already realized at the beginning of 1990s [1, 10, 2022], the continuation approach has been adjusted to the modern, Riemann-Hilbert framework, only recently $[7,19]$. A primary importance of this framework is that it makes possible the rigorous study of the large-time asymptotics via the nonlinear steepest descent method [8].

An alternative approach to the IBV problems with these special boundary conditions (called linearizable) stems from a general approach to IBV problems for integrable nonlinear equations originated by Fokas [11]. The basic idea of this approach consists in considering the both linear equations of the associated Lax pair as spectral problems, each generating respective sets of spectral functions. Consequently, when applied to problems on infinite time intervals (say, $t \in(0, \infty)$ ), this approach requires defining spectral functions associated with the boundary values on these intervals; and thus an information on the large- $t$ behavior of these boundary values is required. The problem here is that, as we have already mentioned above, only a part of boundary values (determining the spectral functions) can be given as boundary conditions; the behavior of the remaining part is unknown and thus has to be provided as an a priori information.

In [19] it has been observed that the Riemann-Hilbert problem obtained in [17] and [15] for linearizable boundary conditions under a priori assumption that the boundary data rapidly decay as $t \rightarrow \infty$ provides the solution of the associated IBV problem without any such assumption, because one can prove independently the validity of the required initial and boundary conditions. The proof goes actually back to the late 1980s works on algebro-geometric solutions of integrable PDEs and is based on the symmetry considerations for the deformed Riemann-Hilbert problem.

All the studies mentioned above are related to the problems with decaying (as $x \rightarrow \infty$ ) initial data. On the other hand, problems (particularly, initial value problems on the whole line) with nondecaying initial data attract a growing interest. As in the case of the decaying boundary conditions, the Riemann-Hilbert approach allows a detailed study of the large-time behavior of solutions. In [5], the initial value (IV) problem for the focusing NLS with generalized "step-like" initial data (vanishing for $x<0$ and periodic in $x(u(x, 0)=\alpha \exp (-2 \mathrm{i} \beta x)$ ) for $x>0)$ is considered and the large-time behavior of the solution is presented.

In [6], the initial value problem for the NLS equations is considered with oscillatory initial data $\alpha \exp (-2 \mathrm{i} \beta|x|)$ with $\beta>0$, which can be viewed as the problem of collision of two plane waves, $\alpha \exp (-2 \mathrm{i} \beta x+\mathrm{i} \omega t))$ and $\alpha \exp (2 \mathrm{i} \beta x+\mathrm{i} \omega t)$ resembling the Toda shock problem [23]. In [6], three regions in space-time with qualitatively different asymptotic behavior of the solution are described, showing, in particular, that for any $x$ fixed, the large- $t$ behavior is described in terms of single phase theta functions.

Notice that in [6], the considered initial data are even w.r.t. $x$, and so is the solution of the IV problem for any $t$. Particularly, this solution satisfies the condition $u_{x}(0, t)=0$ for all $t>0$. Saying differently, the solution $u(x, t)$ of the IV problem in [6] is the even continuation of the solution of 
the initial boundary value problem:

$$
\begin{array}{lr}
\mathrm{i} u_{t}+u_{x x}+2|u|^{2} u=0, & x>0, t>0, \\
u(x, 0)=\alpha \mathrm{e}^{-2 \mathrm{i} \beta x}, & x>0, \\
u_{x}(0, t)=0, & t>0, \\
u(x, t)-u^{p}(x, t) \rightarrow 0 \text { as } x \rightarrow \infty, & t>0,
\end{array}
$$

where $\alpha>0$ and $\beta>0$ are constants, and

$$
u^{p}(x, t)=\alpha \mathrm{e}^{-2 \mathrm{i} \beta x+2 \mathrm{i} \omega t}
$$

with $\omega=\alpha^{2}-2 \beta^{2}$. Notice that $u^{p}(x, t)$ is the exact, plane wave solution of the NLS equation satisfying the initial condition $u^{p}(x, 0)=\alpha \mathrm{e}^{-2 \mathrm{i} \beta x}$.

In the present paper, we consider the initial boundary value problem for the focusing nonlinear Schrödinger equation with oscillatory initial data and the Robin boundary condition:

$$
\begin{array}{lrl}
\mathrm{i} u_{t}+u_{x x}+2|u|^{2} u=0, & x>0, t>0, \\
u(x, 0)=u_{0}(x), & x>0, \\
u_{x}(0, t)+q u(0, t)=0, & t>0, \\
u(x, t)-u^{p}(x, t) \rightarrow 0 \text { as } x \rightarrow \infty, & t>0,
\end{array}
$$

where

$$
u_{0}(x)-\alpha \mathrm{e}^{-2 \mathrm{i} \beta x} \rightarrow 0 \quad \text { as } x \rightarrow+\infty .
$$

Here $\alpha>0$ whereas $\beta \neq 0$ and $q \neq 0$ are real constants which can be positive or negative. To be more specific, a special attention will be paid to the case of pure oscillatory initial data:

$$
u_{0}(x)=\alpha \mathrm{e}^{-2 \mathrm{i} \beta x} \quad \text { for all } x>0 .
$$

Our approach follows the lines of the general approach $[4,17]$ to IBV problems based on the use of the associated Riemann-Hilbert problem (involving the spectral functions associated with the initial condition and the Robin parameter $q$ ) and consists of the following main steps: (i) provide a family of the Riemann-Hilbert problems (parametrized by $x$ and $t$ ) such that the solution $u(x, t)$ of (1.2) is expressed in terms of the solutions of these problems; (ii) prove that $u$ satisfies the initial condition $u(x, 0)=u_{0}(x)$; (iii) prove that $u$ satisfies the boundary condition $u_{x}(0, t)+q u(0, t)=0$. In our approach, item (i) follows the general framework of the simultaneous spectral analysis of the Lax pair equations whereas the proof of (ii) and (iii) are based on appropriate deformations of the original RH problem.

When proving (iii) we deform the $\mathrm{RH}$ problem in such a way that the resulting $\mathrm{RH}$ problem can be viewed as a RH problem associated with an IV problem (on the whole line $-\infty<x<\infty$ ) whose restriction to the half-line $x>0$ gives the solutions of the IBV problem (1.2). Particularly, in the case $u_{0}(x)=\alpha \mathrm{e}^{-2 \mathrm{i} \beta x}$ for $x>0$, we will show that $u(x, 0)=\alpha \mathrm{e}^{\mathrm{i} \phi} \mathrm{e}^{2 \mathrm{i} \beta x}$ for $x<0$, for a certain real phase $\phi$, and thus the IV problem itself can be viewed as a generalized shock problem.

On the other hand, the RH problem formulation allows the detailed study of the large-time asymptotics of the solution of the constructed IV problem as well as of the solution of the original 
IBV problem (1.2). Particularly, the impact of the Robin parameter to the large- $t$ behavior of the solution of the IBV (or IV) problem at $x=0$, observed in the case of the decaying initial conditions, see [19], is present in the case of oscillatory initial conditions as well, namely: in the case $q>0$, the discrete spectrum associated with the IV problem is always non-empty on the imaginary axis, and this generates a breather-type (stationary soliton) oscillatory behavior. Moreover, this breather dominates the large- $t$ behavior of the solution along the $t$-axis in the case $\beta<0$ ("rarefaction wave").

\section{The Riemann-Hilbert formalism for the IBV problem with oscillatory initial data}

In order to adapt the Riemann-Hilbert formalism to IBV problems on the half-line $x \geq 0$ for the NLS equation [17] to the case of non-decaying initial data, we need the eigenfunctions and spectral functions associated with such data [5,6].

Recall that the focusing NLS equation

$$
\mathrm{i} u_{t}+u_{x x}+2|u|^{2} u=0
$$

is the compatibility condition of two linear equations (Lax pair) [24]:

$$
\Psi_{x}+\mathrm{i} k \sigma_{3} \Psi=U \Psi
$$

with

$$
U=\left(\begin{array}{cc}
0 & u \\
-\bar{u} & 0
\end{array}\right)
$$

and

$$
\Psi_{t}+2 \mathrm{i} k^{2} \sigma_{3} \Psi=V \Psi
$$

with

$$
V=\left(\begin{array}{cc}
\mathrm{i}|u|^{2} & 2 k u+\mathrm{i} u_{x} \\
-2 k \bar{u}+\mathrm{i} \bar{u}_{x} & -\mathrm{i}|u|^{2}
\end{array}\right)
$$

Let $U^{p}(x, t):=\left(\begin{array}{cc}0 & u^{p}(x, t) \\ -u^{p}(x, t) & 0\end{array}\right)$ and let $\Psi^{p}(x, t, k)$ be a solution of the Lax pair equations (2.2) and (2.4) with $u \equiv u^{p}$ :

$$
\Psi^{p}(x, t, k)=\mathrm{e}^{\mathrm{i}(\omega t-\beta x) \sigma_{3}} \mathscr{E}(k) \mathrm{e}^{(-\mathrm{i} X(k) x-\mathrm{i} \Omega(k) t) \sigma_{3}},
$$

where

$$
\mathscr{E}(k)=\frac{1}{2}\left(\begin{array}{l}
v(k)+\frac{1}{v(k)} v(k)-\frac{1}{v(k)} \\
v(k)-\frac{1}{v(k)} v(k)+\frac{1}{v(k)}
\end{array}\right)
$$

with

$$
\begin{aligned}
v(k) & =\left(\frac{k-\beta-\mathrm{i} \alpha}{k-\beta+\mathrm{i} \alpha}\right)^{1 / 4}, \\
X(k) & =\sqrt{(k-\beta)^{2}+\alpha^{2}}, \\
\Omega(k) & =2(k+\beta) X(k) .
\end{aligned}
$$


The functions $v(k), X(k)$ and $\Omega(k)$ are defined for $k \in \mathbb{C} \backslash \gamma$, with the branch cut

$$
\gamma=\left\{k \equiv k_{1}+\mathrm{i} k_{2}: k_{1}=\beta, k_{2} \in(-\alpha, \alpha)\right\},
$$

in such a way that $v(k)=1+o(1), X(k)=k-\beta+o(1)$, and $\Omega(k)=2 k^{2}+\omega+o(1)$ as $k \rightarrow \infty$.

Assuming that $u(x, t)$ satisfies (2.1) for $x>0$ and $0 \leq t<T$ with some $T<\infty$ and that $u(x, t)-$ $u^{p}(x, t) \rightarrow 0$ as $x \rightarrow+\infty$ (for all $0 \leq t<T$ ) fast enough, define the solutions $\Psi_{j}(x, t, k), j=1,2,3$ of (2.2)-(2.5) as follows:

$$
\begin{gathered}
\Psi_{j}(x, t, k):=\Phi_{j}(x, t, k) \mathrm{e}^{\left(-\mathrm{i} k x-2 \mathrm{i} k^{2} t\right) \sigma_{3}} \quad \text { for } j=1,2 \\
\Psi_{3}(x, t, k):=\Phi_{3}(x, t, k) \mathrm{e}^{(-\mathrm{i} X(k) x-\mathrm{i} \Omega(k) t) \sigma_{3}}
\end{gathered}
$$

where $\Phi_{j}$ solve the integral equations

$$
\begin{aligned}
\Phi_{1}(x, t, k) & =E-\mathrm{e}^{-\mathrm{i} k x \sigma_{3}} \int_{t}^{T} \mathrm{e}^{-2 \mathrm{i} k^{2}(t-\tau) \sigma_{3}} V(0, \tau, k) \Phi_{1}(0, \tau, k) \mathrm{e}^{2 \mathrm{i} k^{2}(t-\tau) \sigma_{3}} \mathrm{e}^{\mathrm{i} k x \sigma_{3}} \\
& +\int_{0}^{x} \mathrm{e}^{-\mathrm{i} k(x-y) \sigma_{3}} U(y, t) \Phi_{1}(y, t, k) \mathrm{e}^{\mathrm{i} k(x-y) \sigma_{3}} \mathrm{~d} y, \\
\Phi_{2}(x, t, k) & =E+\mathrm{e}^{-\mathrm{i} k x \sigma_{3}} \int_{0}^{t} \mathrm{e}^{-2 \mathrm{i} k^{2}(t-\tau) \sigma_{3}} V(0, \tau, k) \Phi_{2}(0, \tau, k) \mathrm{e}^{2 \mathrm{i} k^{2}(t-\tau) \sigma_{3}} \mathrm{e}^{\mathrm{i} k x \sigma_{3}} \\
& +\int_{0}^{x} \mathrm{e}^{-\mathrm{i} k(x-y) \sigma_{3}} U(y, t) \Phi_{2}(y, t, k) \mathrm{e}^{\mathrm{i} k(x-y) \sigma_{3}} \mathrm{~d} y, \\
\Phi_{3}(x, t, k) & =\mathrm{e}^{\mathrm{i}(\omega t-\beta x) \sigma_{3}} \mathscr{E}(k)-\int_{x}^{\infty} \Gamma^{p}(x, y, t, k)\left[U(y, t)-U^{p}(y, t)\right] \Phi_{3}(y, t, k) \mathrm{e}^{\mathrm{i} X(k)(x-y) \sigma_{3}} \mathrm{~d} y,
\end{aligned}
$$

where $E$ is the $2 \times 2$ identity matrix, with

$$
\Gamma^{p}(x, y, t, k):=\Psi^{p}(x, t, k)\left(\Psi^{p}(y, t, k)\right)^{-1} .
$$

Notice that $\Gamma^{p}(x, y, t, k)$ is an entire function, as the solution of the Cauchy problem $\Gamma_{x}^{p}+\mathrm{i} k \sigma_{3} \Gamma^{p}=$ $U^{p} \Gamma^{p}, \Gamma^{p}(y)=E$.

From (2.2) and (2.4) it follows that $\operatorname{det} \Phi_{j}=\operatorname{det} \Psi_{j} \equiv 1$ for all $k$ where the corresponding $\Phi_{j}(k)$ is defined. From (2.9) it follows that $\Phi_{1}(\cdot, \cdot, k)$ and $\Phi_{2}(\cdot, \cdot, k)$ are entire in $k$ whereas $\Phi_{3}(\cdot, \cdot, k)$ is columnwise analytic: the first column $\Phi_{3}^{(1)}(\cdot, \cdot, k)$ of $\Phi_{3}$ is analytic in $\mathbb{C}_{-} \backslash \gamma$ and the second column $\Phi_{3}^{(2)}(\cdot, \cdot, k)$ is analytic in $\mathbb{C}_{+} \backslash \gamma$. In what follows, we will denote by $f_{ \pm}(k), k \in \gamma$ the limiting values of a function $f(k)$ as $k$ approaches $\gamma$ from the left $(+)$ or from the right $(-)$.

Besides, the whole matrices $\left(\Phi_{3}\right)_{ \pm}(\cdot, \cdot, k)$ are determined for $k$ on $\gamma_{ \pm}$, the corresponding side of $\gamma$. In order to describe them (or $\left(\Psi_{3}\right)_{ \pm}(\cdot, \cdot, k)$ ), it is convenient to introduce the $2 \times 2$ function $\mu(x, t, k), k \in \gamma$ as the solution of the integral equation

$$
\mu(x, t, k)=E-\int_{x}^{\infty} \Gamma^{p}(x, y, t, k)\left[U(y, t)-U^{p}(y, t)\right] \mu(y, t, k)\left(\Gamma^{p}\right)^{-1}(x, y, t, k) \mathrm{d} y,
$$

in terms of which we have

$$
\Psi_{3}(x, t, k)=\mu(x, t, k) \Psi^{p}(x, t, k)
$$

Thus

$$
\left(\Psi_{3}\right)_{ \pm}(x, t, k)=\mu(x, t, k) \Psi_{ \pm}^{p}(x, t, k), \quad k \in \gamma
$$


Now define the scattering matrices $s(k)$ and $S(k), k \in \mathbb{R} \cup \gamma_{+} \cup \gamma_{-}$, as the matrices relating the eigenfunctions $\Psi_{j}(x, t, k)$ for all $x$ and $t$ :

$$
\Psi_{3}(x, t, k)=\Psi_{2}(x, t, k) s(k), \quad \Psi_{1}(x, t, k)=\Psi_{2}(x, t, k) S(k) .
$$

From (2.9) and (2.11) it follows that

$$
s(k)=\Psi_{3}(0,0, k)
$$

Thus the the symmetry

$$
\overline{\left(\Psi_{j}\right)_{11}(x, t, \bar{k})}=\left(\Psi_{j}\right)_{22}(x, t, k), \quad \overline{\left(\Psi_{j}\right)_{12}(x, t, \bar{k})}=-\left(\Psi_{j}\right)_{21}(x, t, k)
$$

implies that

$$
s(k)=\left(\begin{array}{cc}
\bar{a}(k) & b(k) \\
-\bar{b}(k) & a(k)
\end{array}\right), \quad S(k)=\left(\begin{array}{cc}
\bar{A}(k) & B(k) \\
-\bar{B}(k) & A(k)
\end{array}\right), \quad k \in \mathbb{R} .
$$

Moreover, $\operatorname{det} s(k)=\operatorname{det} S(k)=1$.

It follows from the definition of $a(k)$ and $b(k)$ and the analyticity properties of $\Psi_{3}$ that the spectral functions $a(k)$ and $b(k)$ are analytic in $k \in \mathbb{C}^{+} \backslash \gamma$; moreover, $a(k) \rightarrow 1$ and $b(k) \rightarrow 0$ as $k \rightarrow \infty$, and the limiting values $a_{ \pm}(k)$ and $b_{ \pm}(k), k \in \gamma$ are related as follows:

\section{Lemma 2.1.}

$$
a_{-}(k) b_{+}(k)-a_{+}(k) b_{-}(k)=\mathrm{i}, \quad k \in \gamma .
$$

Proof. Indeed, the representation (2.10) yields

$$
s_{ \pm}(k)=\mu(0,0, k) \mathscr{E}_{ \pm}(k) .
$$

From (2.7) and (2.8) we have $v_{+}(k)=\mathrm{i} v_{-}(k)$ and thus $\mathscr{E}_{+}(k)=\mathrm{i} \mathscr{E}_{-}(k)\left(\begin{array}{ll}0 & 1 \\ 1 & 0\end{array}\right)$, which yields

$$
s_{+}(k)=\mathrm{i} s_{-}(k)\left(\begin{array}{ll}
0 & 1 \\
1 & 0
\end{array}\right)
$$

In terms of $a_{ \pm}$and $b_{ \pm}$this reads

$$
s_{+}(k)=\left(\begin{array}{cc}
\mathrm{i} b_{-}(k) & b_{+}(k) \\
\mathrm{i} a_{-}(k) & a_{+}(k)
\end{array}\right), \quad s_{-}(k)=\left(\begin{array}{cc}
-\mathrm{i} b_{+}(k) & b_{-}(k) \\
-\mathrm{i} a_{+}(k) & a_{-}(k)
\end{array}\right) .
$$

Finally, the determinant relation $\operatorname{det} s \equiv 1$ gives (2.14).

In the particular case of pure exponential initial data, $u(x, 0)=\alpha \mathrm{e}^{-2 \mathrm{i} \beta x}$ for $x>0$, we have $s(k)=\Psi_{3}(0,0, k)=\mathscr{E}(k)$ and thus

$$
\begin{aligned}
& a(k)=\overline{a(\bar{k})}=\frac{1}{2}\left(v(k)+\frac{1}{v(k)}\right), \\
& b(k)=-\overline{b(\bar{k})}=\frac{1}{2}\left(v(k)-\frac{1}{v(k)}\right) .
\end{aligned}
$$

In this case, the identity (2.14) is seen directly. 
The spectral functions $A=A(k ; T)$ and $B=B(k ; T)$ defined by (2.13) and (2.11) in terms of $\Psi_{1}(0,0, k)$, are entire functions bounded in the domains $I$ and $I I I$, where $I=\{k: \operatorname{Im} k>0, \operatorname{Re} k>0\}$ and $I I I=\{k: \operatorname{Im} k<0, \operatorname{Re} k<0\}$, with $A(k ; T) \rightarrow 1$ and $B(k ; T) \rightarrow 0$ as $k \rightarrow \infty$. Moreover, they are determined by $u(0, t)$ and $u_{x}(0, t)$ for $0 \leq t \leq T$ only, via

$$
S(k ; T)=\left(\Psi_{2}\right)^{-1}(0, t, k) \Psi_{1}(0, t, k)
$$

for any $t \in[0, T]$.

The compatibility of the set of functions $\left\{u(x, 0), u(0, t), u_{x}(0, t)\right\}$ as traces of a solution $u(x, t)$ of the NLS equation can be expressed in terms of the associated spectral functions as follows [17]:

$$
A(k ; T) b(k)-a(k) B(k ; T)=c(k ; T) \mathrm{e}^{4 \mathrm{i} k^{2} T}, \quad k \in \mathbb{C}^{+} \backslash \gamma,
$$

with some $c(k ; T)=O\left(\frac{1}{k}\right)$ as $k \rightarrow \infty$ (in the general scheme [12] of analysis of IBV problems, (2.16) is called the global relation).

Define

$$
d(k):=a(k) \overline{A(\bar{k})}+b(k) \overline{B(\bar{k})}, \quad k \in I I \backslash \gamma,
$$

where $I I=\{k: \operatorname{Im} k>0, \operatorname{Re} k<0\}$. Finally, assuming that $d(k)$ has at most a finite number of simple zeros in $I I$, define a piecewise meromorphic function (the superscripts denote the column of the respective matrix) $M(x, t, k), k \in \mathbb{C} \backslash\{\mathbb{R} \cup \mathrm{i} \mathbb{R} \cup \gamma\}$ :

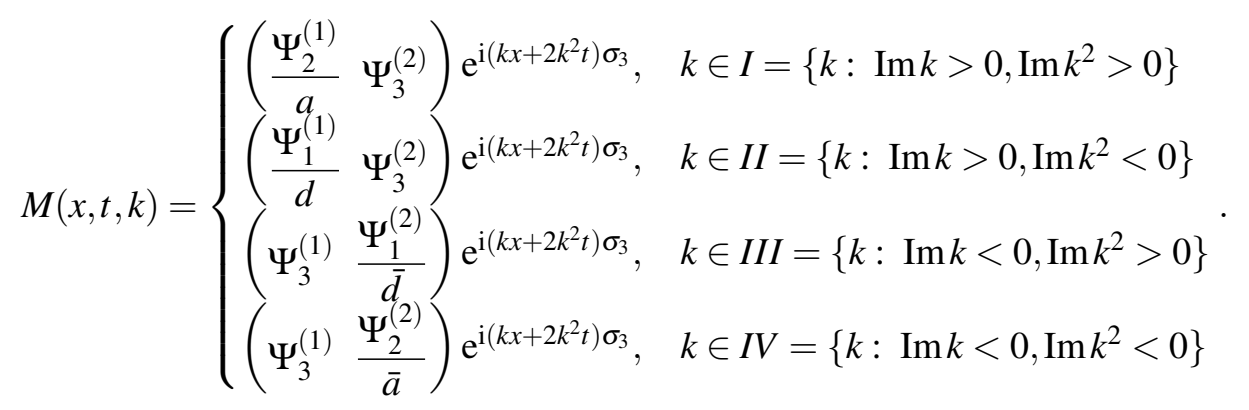

Then $M(\cdot, \cdot, k)=E+O(1 / k)$ as $k \rightarrow \infty$, and the scattering relations (2.11) imply that the limiting values of $M$ on $\mathbb{R} \cup i \mathbb{R} \cup \gamma$ satisfy the jump relations

$$
M_{+}(x, t, k)=M_{-}(x, t, k) \mathrm{e}^{-\mathrm{i}\left(k x+2 k^{2} t\right) \sigma_{3}} J_{0}(k) \mathrm{e}^{\mathrm{i}\left(k x+2 k^{2} t\right) \sigma_{3}},
$$

where $J_{0}(k)$ is defined as follows.

(1) For $k \in \mathbb{R} \cup \mathrm{i} \mathbb{R}$,

$$
J_{0}(k):= \begin{cases}\left(\begin{array}{cc}
1+|r(k)|^{2} \bar{r}(k) \\
r(k) & 1
\end{array}\right), & k>0, \\
\left(\begin{array}{cc}
1 & 0 \\
\Gamma(k) & 1
\end{array}\right), & k \in \mathbb{R}_{+}, \\
\left(\begin{array}{cc}
1 \bar{\Gamma}(\bar{k}) \\
0 & 1
\end{array}\right), & k \in \mathbb{R}_{-}, \\
\left(\begin{array}{cc}
1+|r(k)+\Gamma(k)|^{2} \bar{r}(k)+\bar{\Gamma}(k) \\
r(k)+\Gamma(k) & 1
\end{array}\right), & k<0,\end{cases}
$$


where

$$
r(k):=\frac{\bar{b}(k)}{a(k)}, \quad \Gamma(k):=-\frac{\bar{B}(\bar{k})}{a(k) d(k)} \equiv-\frac{\bar{B}(\bar{k}) / \bar{A}(\bar{k})}{a(k)(a(k)+b(k) \bar{B}(\bar{k}) / \bar{A}(\bar{k}))} .
$$

Here the orientation of the contour is chosen as from $-\infty$ to $+\infty$ along $\mathbb{R}$ and away from 0 along $i \mathbb{R}$.

(2) For $k \in \gamma$, with the orientation of $\gamma$ upward, the definition of $J_{0}(k)$ depends on the sign of $\beta$ :

- if $\beta>0$, then $\gamma \subset I \cup I V$, and

$$
J_{0}(k):= \begin{cases}\left(\begin{array}{cc}
\frac{a_{-}(k)}{a_{+}(k)} \Gamma_{1}(k) \\
0 & \frac{a_{+}(k)}{a_{-}(k)}
\end{array}\right), & k \in \gamma \cap I, \\
\left(\begin{array}{cc}
\bar{a}_{+}(\bar{k}) & 0 \\
\bar{a}_{-}(\bar{k}) & 0 \\
-\bar{\Gamma}_{1}(\bar{k}) & \frac{\bar{a}_{-}(\bar{k})}{\bar{a}_{+}(\bar{k})}
\end{array}\right), & k \in \gamma \cap I V\end{cases}
$$

with

$$
\Gamma_{1}(k):=b_{+}(k) a_{-}(k)-b_{-}(k) a_{+}(k) .
$$

Taking into account (2.14), we have $\Gamma_{1}(k) \equiv \mathrm{i}$ and thus the jump (2.10) takes the form

$$
J_{0}(k)=\left\{\begin{array}{cc}
\left(\begin{array}{cc}
\frac{a_{-}(k)}{a_{+}(k)} & \mathrm{i} \\
0 & \frac{a_{+}(k)}{a_{-}(k)}
\end{array}\right), & k \in \gamma \cap I, \\
\left(\begin{array}{cc}
\bar{a}_{+}(\bar{k}) & 0 \\
\bar{a}_{-}(\bar{k}) & 0 \\
\mathrm{i} & \frac{\bar{a}_{-}(\bar{k})}{\bar{a}_{+}(\bar{k})}
\end{array}\right), & k \in \gamma \cap I V
\end{array}\right.
$$

- if $\beta<0$, then $\gamma \subset I I \cup I I I$, and

$$
J_{0}(k):= \begin{cases}\left(\begin{array}{cc}
\frac{d_{-}(k)}{d_{+}(k)} \hat{\Gamma}_{1}(k) \\
0 & d_{+}(k) \\
d_{-}(k)
\end{array}\right), & k \in \gamma \cap I I, \\
\left(\begin{array}{cc}
\bar{d}_{+}(\bar{k}) & 0 \\
\bar{d}_{\bar{t}}(\bar{k}) & 0 \\
-\overline{\hat{\Gamma}}_{1}(\bar{k}) & \bar{d}_{-}(\bar{k}) \\
\bar{d}_{+}(\bar{k})
\end{array}\right), & k \in \gamma \cap I I I\end{cases}
$$

with $\hat{\Gamma}_{1}(k):=\left(A(k) b_{+}(k)-B(k) a_{+}(k)\right) d_{-}(k)-\left(A(k) b_{-}(k)-B(k) a_{-}(k)\right) d_{+}(k)$.

Direct calculations, taking into account the determinant equality $A(k) \bar{A}(\bar{k})+B(k) \bar{B}(\bar{k})=1$ (coming from $\operatorname{det} S \equiv 1$ ) show that $\hat{\Gamma}_{1}(k)=\Gamma_{1}(k)$ and thus $\hat{\Gamma}_{1}(k) \equiv \mathrm{i}$, which yields

$$
J_{0}(k)=\left\{\begin{array}{cl}
\left(\begin{array}{cc}
\frac{d_{-}(k)}{d_{+}(k)} & \mathrm{i} \\
0 & \frac{d_{+}(k)}{d_{-}(k)}
\end{array}\right), & k \in \gamma \cap I I, \\
\left(\begin{array}{cc}
\frac{\bar{d}_{+}(\bar{k})}{\bar{d}_{-}(\bar{k})} & 0 \\
\mathrm{i} & \frac{\bar{d}_{-}(\bar{k})}{\bar{d}_{+}(\bar{k})}
\end{array}\right), & k \in \gamma \cap I I I .
\end{array}\right.
$$


Complemented with the normalization condition $M=E+O(1 / k)$ as $k \rightarrow \infty$ and the respective residue conditions at the zeros of $d(k)$ and $a(k)$ (see [17] for details), the jump relation (2.19) can be viewed as the Riemann-Hilbert problem: given $\{a(k), b(k), A(k), B(k)\}$ (and the residue parameters, if any), find $M(x, t, k)$ for all $x \geq 0$ and $t \geq 0$. Then the solution of the NLS equation, $u(x, t)$, is given in terms of $M(x, t, k)$ by

$$
u(x, t)=2 \mathrm{i} \lim _{k \rightarrow \infty} k M_{12}(x, t, k) .
$$

Moreover, $u(x, 0)$ generates $\{a(k), b(k)\}$ and $\left\{u(0, t), u_{x}(0, t)\right\}$ generates $\{A(k), B(k)\}$ as the corresponding spectral functions provided the latter verify the global relation (2.16). Therefore, the Riemann-Hilbert problem approach gives the solution of the overdetermined IBV problem

$$
\begin{array}{lr}
\mathrm{i} u_{t}+u_{x x}+2|u|^{2} u=0, & x>0, t>0, \\
u(x, 0)=u_{0}(x), & x \geq 0, \\
u(0, t)=v_{0}(t), & 0 \leq t \leq T, \\
u_{x}(0, t)=v_{1}(t), & 0 \leq t \leq T
\end{array}
$$

provided that the spectral functions $\{a(k), b(k), A(k), B(k)\}$ constructed from $\left\{u_{0}(x), v_{0}(t), v_{1}(t)\right\}$ satisfy the global relation (2.16).

\section{The Riemann-Hilbert formalism for Robin boundary condition}

The overdetermined nature of the Riemann-Hilbert problem approach to the IBV problems can be overcome in certain special cases of boundary conditions [12]. An efficient way to do this is the use of an additional symmetry in the spectral problem for the $t$-equation of the Lax pair.

Since the $t$-equation, considered alone, is independent of the initial conditions, the following symmetry considerations, associated with the Robin boundary condition, are exactly the same as in $[17,19]$, where the IBV with decaying initial conditions were considered. Namely, if $u+q u_{x}=0$ with some $q \in \mathbb{R}$, then the matrix $\tilde{V}:=V-2 \mathrm{i} k^{2} \sigma_{3}$ of the $t$-equation $\Psi_{t}=\tilde{V} \Psi$ satisfies the symmetry relation [17]

$$
\tilde{V}(x, t,-k)=N(k) \tilde{V}(x, t, k) N^{-1}(k)
$$

where $N(k)=\operatorname{diag}\left\{N_{1}(k), N_{2}(k)\right\}$ with $N_{1}(k)=2 k+\mathrm{i} q$ and $N_{2}(k)=-2 k+\mathrm{i} q$. In turn, (3.1) implies the symmetry for $S: S(-k ; T)=N(k) S(k ; t) N^{-1}(k)$, which reads in terms of $A$ and $B$ as

$$
\begin{aligned}
& A(-k ; T)=A(k ; T), \\
& B(-k ; T)=-\frac{2 k+\mathrm{i} q}{2 k-\mathrm{i} q} B(k ; T) .
\end{aligned}
$$

Now we observe that the global relation (2.16) also has the same form as in the case of decaying initial conditions, which, being combined with the symmetry relation (3.2), suggests rewriting the $\mathrm{RH}$ problem (2.19) in the form that uses only the spectral functions $a(k)$ and $b(k)$ associated with the initial values $u(x, 0)$. Namely, since the exponential in the r.h.s. of (2.16) is rapidly decaying for $k \in I$, the global relation (2.16) suggests to replace $\frac{B}{A}(k ; T)$ by $\frac{b}{a}(k)$ for $k \in I$. Then, the symmetry 
relation (3.2) suggests to replace

$$
\frac{B}{A}(k ; T) \mapsto-\frac{2 k-\mathrm{i} q}{2 k+\mathrm{i} q} \frac{b}{a}(-k) \quad \text { for } k \in I I I
$$

and consequently to replace

$$
\frac{\bar{B}}{\bar{A}}(\bar{k} ; T) \mapsto-\frac{2 k+\mathrm{i} q}{2 k-\mathrm{i} q} \overline{\bar{a}}(-\bar{k}) \quad \text { for } k \in I I .
$$

Remark 3.1. We emphasize that we are not going to compare the RH problem obtained as a result of the replacement described above, with the original RH problem (2.19)-(2.26). Instead, we will show directly that the resulting RH problem (for $\hat{M}$, see below) gives the solution of the IBV problem (1.2).

Remark 3.2. A principal difference when comparing with the case of decaying initial conditions is that now $a(k)$ and $b(k)$ (as well as their ratio) has jump discontinuities across $\gamma$, which affects the jump conditions on $\gamma$ and eventually on $\gamma_{1}$, where $\gamma_{1}$ is symmetric to $\gamma$ with respect to the imaginary axis:

$$
\gamma_{1}:=\left\{k=k_{1}+\mathrm{i} k_{2}: k_{1}=-\beta, k_{2} \in(-\alpha, \alpha)\right\}
$$

(the latter is due to the jump of $\bar{a}(-\bar{k})$ and $\bar{b}(-\bar{k})$ across $\gamma_{1}$ ).

The resulting jump conditions

$$
\tilde{M}_{+}(x, t, k)=\tilde{M}_{-}(x, t, k) \mathrm{e}^{-\mathrm{i}\left(k x+2 k^{2} t\right) \sigma_{3}} \tilde{J}_{0}(k) \mathrm{e}^{\mathrm{i}\left(k x+2 k^{2} t\right) \sigma_{3}},
$$

are as follows.

(1) For $k \in \mathbb{R} \cup \mathrm{i} \mathbb{R}, \tilde{J_{0}}(k)$ has the same form as in (2.20), but with $\Gamma(k)$ replaced, accordingly to (2.21) and (3.4), by $\tilde{\Gamma}(k)$ (cf. [17, 19]), where

$$
\begin{aligned}
& \tilde{\Gamma}(k):=\frac{\overline{b(-\bar{k})}}{a(k)} \frac{2 k+\mathrm{i} q}{(2 k-\mathrm{i} q) a(k) \overline{a(-\bar{k})}-(2 k+\mathrm{i} q) b(k) \overline{b(-\bar{k})}}: \\
& \tilde{J}_{0}(k):= \begin{cases}\left(\begin{array}{cc}
1+|r(k)|^{2} & \bar{r}(k) \\
r(k) & 1
\end{array}\right), & k>0, \\
\left(\begin{array}{cc}
1 & 0 \\
\tilde{\Gamma}(k) & 1
\end{array}\right), & k \in \mathbb{R}_{+}, \\
\left(\begin{array}{cc}
1 & \tilde{\Gamma}(\bar{k}) \\
0 & 1
\end{array}\right), & k \in \mathbb{R}_{-}, \\
\left(\begin{array}{cc}
1+\left|r_{e}(k)\right|^{2} & \bar{r}_{e}(k) \\
r_{e}(k) & 1
\end{array}\right), & k<0,\end{cases}
\end{aligned}
$$

and

$$
r_{e}(k):=r(k)+\tilde{\Gamma}(k)=\frac{(2 k-\mathrm{i} q) \overline{b(k) a(-k)}+(2 k+\mathrm{i} q) \overline{b(-k) a(k)}}{(2 k-\mathrm{i} q) a(k) \overline{a(-k)}-(2 k+\mathrm{i} q) \overline{b(-k)} b(k)}
$$


(2) For $k \in \gamma$, the jump for $\tilde{M}$ depends on the sign of $\beta$.

- if $\beta>0$, then $\tilde{J}_{0}(k)=J_{0}(k)$, see (2.24) (notice that in this case, $J_{0}(k)$ is already defined in terms of $a(k)$ and $b(k)$ only).

- if $\beta<0$, then $\gamma \in I I \cup I I I$, and

$$
\frac{d_{-}(k)}{d_{+}(k)} \equiv \frac{a_{-}(k)+b_{-}(k) \frac{\bar{B}(\bar{k})}{\bar{A}(\bar{k})}}{a_{+}(k)+b_{+}(k) \frac{\bar{B}(\bar{k})}{\bar{A}(\bar{k})}}
$$

is replaced, accordingly to (3.4), by

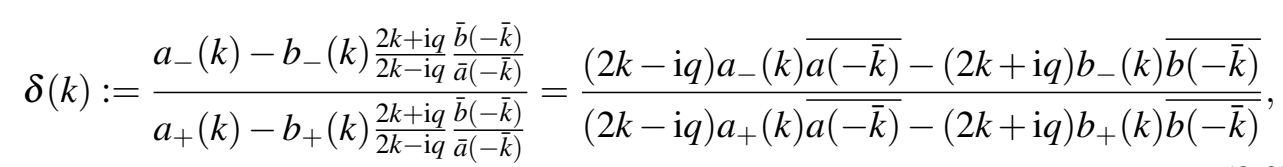

which leads to

$$
\tilde{J}_{0}(k)=\left\{\begin{array}{cc}
\left(\begin{array}{cc}
\delta(k) & \mathrm{i} \\
0 & \delta^{-1}(k)
\end{array}\right), & k \in \gamma \cap \mathbb{C}_{+}, \\
\left(\begin{array}{cc}
\bar{\delta}^{-1}(\bar{k}) & 0 \\
\mathrm{i} & \bar{\delta}(\bar{k})
\end{array}\right), & k \in \gamma \cap \mathbb{C}_{-}
\end{array}\right.
$$

(3) In the case $\beta>0$, the fact that $\tilde{\Gamma}(k)$ has an additional (with respect to $\Gamma(k)$ ) jump across $\gamma_{1}$ suggests introducing the jump conditions for $\tilde{M}$ on $\gamma_{1}$ :

$$
\tilde{J}_{0}(k)=\left\{\begin{array}{cc}
\left(\begin{array}{cr}
1 & 0 \\
\tilde{\Gamma}_{+}(k)-\tilde{\Gamma}_{-}(k) & 1
\end{array}\right), & k \in \gamma_{1} \cap \mathbb{C}_{+} \\
\left(\begin{array}{c}
1 \tilde{\Gamma}_{-}(k)-\tilde{\Gamma}_{+}(k) \\
0 \\
1
\end{array}\right), & k \in \gamma_{1} \cap \mathbb{C}_{-}
\end{array}\right.
$$

(actually, in this case $\gamma_{1} \subset I I \cup I I I$ whereas $\gamma \subset I \cup I V$ ).

At this stage, the jump conditions in the cases $\beta>0$ and $\beta<0$ look quite different. But now we notice that the exponentials in $\left(\begin{array}{cc}1 & 0 \\ \tilde{\Gamma}(k) \mathrm{e}^{2 \mathrm{i} k x+4 \mathrm{i} k^{2} t} & 1\end{array}\right)$ and $\left(\begin{array}{cc}1 & \tilde{\Gamma}(\bar{k}) \mathrm{e}^{-2 \mathrm{i} k x-4 \mathrm{i} k^{2} t} \\ 0 & 1\end{array}\right)$ are bounded in respectively $I$ and $I V$. Therefore, we can deform the RH problem with jump (3.5) on $\mathbb{R} \cup \mathrm{i} \mathbb{R} \cup \gamma \cup \gamma_{1}$ to that on $\mathbb{R} \cup \gamma \cup \gamma_{1}$ (thus getting rid of the jump on $i \mathbb{R}$ ) by introducing

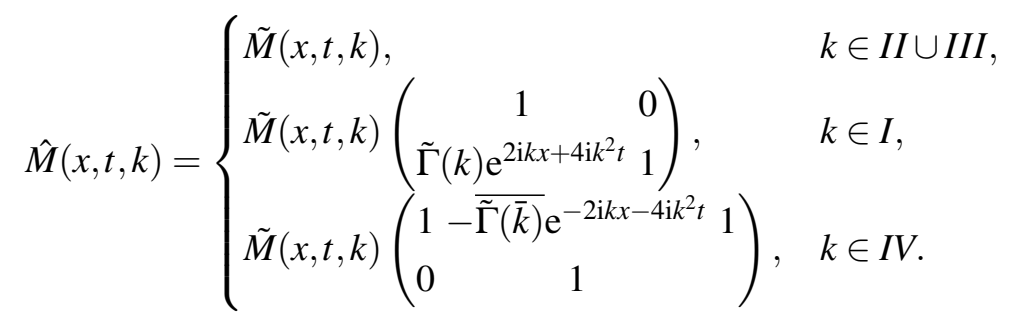

The resulting jump conditions take the form

$$
\hat{M}_{+}(x, t, k)=\hat{M}_{-}(x, t, k) \mathrm{e}^{-\mathrm{i}\left(k x+2 k^{2} t\right) \sigma_{3}} \hat{J}_{0}(k) \mathrm{e}^{\mathrm{i}\left(k x+2 k^{2} t\right) \sigma_{3}}, \quad k \in \mathbb{R} \cup \gamma \cup \gamma_{1},
$$

where: 
(1) for $k \in \mathbb{R}$,

$$
\hat{J}_{0}(k)=\left(\begin{array}{cc}
1+\left|r_{e}(k)\right|^{2} & \bar{r}_{e}(k) \\
r_{e}(k) & 1
\end{array}\right)
$$

with $r_{e}(k)$ defined by (3.8).

(2)

- in the case $\beta>0$, direct calculations give

$$
\hat{J}_{0}(k)=\left(\begin{array}{cc}
1 & 0 \\
-\tilde{\Gamma}_{-}(k) & 1
\end{array}\right)\left(\begin{array}{cc}
\frac{a_{-}(k)}{a_{+}(k)} & \mathrm{i} \\
0 & \frac{a_{+}(k)}{a_{-}(k)}
\end{array}\right)\left(\begin{array}{cc}
1 & 0 \\
\tilde{\Gamma}_{+}(k) & 1
\end{array}\right)=\left(\begin{array}{cc}
\delta(k) & \mathrm{i} \\
0 & \delta^{-1}(k)
\end{array}\right) \quad \text { for } k \in \gamma \cap I
$$

and

$$
\hat{J}_{0}(k)=\left(\begin{array}{cc}
1 & \bar{\Gamma}_{-}(\bar{k}) \\
0 & 1
\end{array}\right)\left(\begin{array}{cc}
\frac{\bar{a}_{+}(\bar{k})}{\bar{a}_{-}(\bar{k})} & 0 \\
\mathrm{i} & \frac{\bar{a}_{-}(\bar{k})}{\bar{a}_{+}(\bar{k})}
\end{array}\right)\left(\begin{array}{cc}
1 & -\overline{\tilde{\Gamma}}_{+}(\bar{k}) \\
0 & 1
\end{array}\right)=\left(\begin{array}{cc}
\bar{\delta}^{-1}(\bar{k}) & 0 \\
\mathrm{i} & \bar{\delta}(\bar{k})
\end{array}\right) \quad \text { for } k \in \gamma \cap I V
$$

and thus the jump takes the form of (3.10) as in the case $\beta<0$. On the other hand, the jump is not changed for $k \in \gamma_{1}: \hat{J}_{0}(k)=\tilde{J}_{0}(k)$ as in (3.11).

- in the case $\beta<0$, a new jump occurs on $\gamma_{1}$, due to the jump of $\tilde{\Gamma}$, having the form (3.11) as in the case $\beta>0$ :

$$
\tilde{J}_{0}(k)=\left\{\begin{array}{cl}
\left(\begin{array}{cr}
1 & 0 \\
\tilde{\Gamma}_{+}(k)-\tilde{\Gamma}_{-}(k) & 1
\end{array}\right), & k \in \gamma_{1} \cap I \\
\left(\begin{array}{c}
1 \tilde{\Gamma}_{-}(k)-\tilde{\tilde{\Gamma}}_{+}(k) \\
0 \\
1
\end{array}\right), & k \in \gamma_{1} \cap I V
\end{array} .\right.
$$

On the other hand, the jump is not changed for $k \in \gamma: \hat{J}_{0}(k)=\tilde{J}_{0}(k)$ as in (3.10).

Summarizing, in the both cases, $\beta>0$ and $\beta<0$, we arrive at

Proposition 3.1. Assume that $u(x, t)$ is the solution of the IBV problem (1.2). Then

$$
u(x, t)=2 \mathrm{i} \lim _{k \rightarrow \infty} k \hat{M}_{12}(x, t, k),
$$

where $\hat{M}$ is a piece-wise meromorphic function satisfying jump conditions (3.13). The jump matrix is given by (3.14) for $k \in \mathbb{R}$ and by $\hat{J}(k)=\tilde{J}(k)$ for $k \in \gamma \cup \gamma_{1}$, where $\tilde{J}(k)$ is defined in terms of $a(k), b(k)$ and $q$ by (3.10) and (3.11) taking into account (3.6) and (3.9). Here $a(k)$ and $b(k)$ are determined by the initial values $u(x, 0)=u_{0}(x), x>0$ through

$$
\left(\begin{array}{l}
b(k) \\
a(k)
\end{array}\right)=\Psi_{3}^{(2)}(0,0, k)=\Phi_{3}^{(2)}(0,0, k)
$$

where $\Phi_{3}^{(2)}(x, 0, k)$ is the solution of $(2.9 c)$ with $U(y)=\left(\begin{array}{cc}0 & u_{0}(y) \\ \bar{u}_{0}(y) & 0\end{array}\right)$, see (2.3).

If the denominator in (3.8) has no zeros in $\mathbb{C}^{+}$, then $\hat{M}$ is uniquely determined as the solution of the Riemann-Hilbert (RH) problem: given $a(k), b(k)$ and $q$, find a piecewise analytic (relative to $\mathbb{R} \cup \gamma \cup \gamma_{1}$ ), matrix-valued function satisfying the jump conditions from Proposition 3.1 and the normalization condition $\hat{M} \rightarrow E$ as $k \rightarrow \infty$. In case the denominator in (3.8) has zeros, the formulation of 
the RH problem is to be complemented by the residue conditions at these points. Introducing $a_{e}(k)$ by (3.25), see below, and assuming that the zeros $k_{j}, j=1, \ldots, N$ of $a_{e}(k)\left(\operatorname{Im} k_{j}>0\right)$ are simple and do not coincide with possible zeros of $a(k)$, the residue conditions are as follows (cf. [17]):

$$
\begin{aligned}
& \operatorname{Res}_{k_{j}} \hat{M}^{(1)}(x, t, k)=\frac{\overline{b\left(-\bar{k}_{j}\right)} \mathrm{e}^{2 \mathrm{i} \Theta\left(x, t, k_{j}\right)}}{\dot{a}_{e}\left(k_{j}\right) a\left(k_{j}\right)} \chi\left(k_{j}\right) \hat{M}^{(2)}\left(x, t, k_{j}\right), \\
& \operatorname{Res}_{\bar{k}_{j}} \hat{M}^{(2)}(x, t, k)=\frac{b\left(-\bar{k}_{j}\right) \mathrm{e}^{-2 \mathrm{i} \bar{\Theta}\left(x, t, k_{j}\right)}}{\overline{\dot{a}_{e}\left(k_{j}\right) a\left(k_{j}\right)}} \bar{\chi}\left(k_{j}\right) \hat{M}^{(1)}\left(x, t, \bar{k}_{j}\right),
\end{aligned}
$$

where $\Theta(x, t, k)=k x+2 k^{2} t$ and

$$
\chi(k)= \begin{cases}\frac{2 k+\mathrm{i} q}{2 k-\mathrm{i} q}, & \text { case } 1 \\ 1, & \text { case } 2\end{cases}
$$

(for the definition of cases 1 and 2, see (3.25) and (3.26) below).

Comparing this with the case of the Robin IBV problem with decaying initial data [19], the RH problem in the oscillatory case has two additional jumps, across $\gamma$ and $\gamma_{1}$. On the other hand, it has the same structure as in the case of the shock IV problem [6], which of course is not surprising because, as we discussed in Introduction, the shock IV problem in [6] corresponds to $q=0$ in (1.2).

Now, having the RH problem formulated in terms of the spectral functions $a(k)$ and $b(k)$ associated with the initial data in (1.2) and of the Robin constant $q$, we can prove directly (without making reference to the boundary values and the spectral functions $A(k)$ and $B(k)$ associated with them) the following Theorem (we formulate it for the case of pure exponential initial data in (1.2); for the general case, see Remark 3.3 below):

Theorem 3.1. Let $\hat{M}(x, t, k)$ be the solution of the Riemann-Hilbert problem with the jump conditions (3.13) and residue conditions (3.17) determined in terms $q$ and the spectral functions $a(k)$ and $b(k)$ (2.15) via (3.8)-(3.14). Let $u(x, t)$ be determined in terms of $\hat{M}(x, t, k)$ via (3.16). Then $u(x, t)$ is a solution of the IBV problem (1.2) with $u_{0}(x)=\alpha \mathrm{e}^{-2 \mathrm{i} \beta x}$.

Proof. (i) The function determined by (3.16) via the solution $\hat{M}$ of the RH problem satisfies the NLS equation in the domain $x>0, t>0$; this is a standard fact based on ideas of the dressing method, see, e.g., [9].

(ii) In order to verify the initial condition $u(x, 0)=u_{0}(x)$, one observes that for $t=0$ and $x>0$, the exponentials in $\left(\begin{array}{cc}1 & 0 \\ \tilde{\Gamma}(k) \mathrm{e}^{2 \mathrm{i} k x} & 1\end{array}\right)$ and $\left(\begin{array}{cc}1 & \tilde{\Gamma}(\bar{k}) \mathrm{e}^{-2 \mathrm{i} k x} \\ 0 & 1\end{array}\right)$ are bounded in respectively $\mathbb{C}_{+}$and $\mathbb{C}_{-}$. Thus we can deform the RH problem with jump (3.13) (for $t=0$ ) as follows: define $\check{M}(x, 0, k)$ by

$$
\check{M}(x, 0, k)=\left\{\begin{array}{cl}
\hat{M}(x, 0, k)\left(\begin{array}{cc}
1 & 0 \\
-\tilde{\Gamma} \mathrm{e}^{2 \mathrm{i} k x} & 1
\end{array}\right), & k \in \mathbb{C}_{+}, \\
\hat{M}(x, 0, k)\left(\begin{array}{cc}
1 & \tilde{\Gamma} \mathrm{e}^{-2 \mathrm{i} k x} \\
0 & 1
\end{array}\right), & k \in \mathbb{C}_{-} .
\end{array}\right.
$$

This makes (i) the jump across $\gamma_{1}$ to disappear: $\check{M}_{+}(x, 0, k)=\check{M}_{-}(x, 0, k), k \in \gamma_{1}$; (ii) the residue conditions to disappear as well (since in the case of (2.15), $a(k)$ has no zeros). On the other hand, the jumps across $\mathbb{R}$ and $\gamma$ take the form 


$$
\check{M}_{+}(x, 0, k)=\check{M}_{-}(x, 0, k) \mathrm{e}^{-\mathrm{i} k x \sigma_{3}} \check{J}_{0}(k) \mathrm{e}^{\mathrm{i} k x \sigma_{3}}, \quad k \in \mathbb{R} \cup \gamma,
$$

where

$$
\check{J_{0}}(k)= \begin{cases}\left(\begin{array}{cc}
1+|r(k)|^{2} \bar{r}(k) \\
r(k) & 1
\end{array}\right), & k \in \mathbb{R}, \\
\left(\begin{array}{cc}
\frac{a_{-}(k)}{a_{+}(k)} \Gamma_{1}(k) \\
0 & \frac{a_{+}(k)}{a_{-}(k)}
\end{array}\right), & k \in \gamma \cap \mathbb{C}_{+}, . \\
\left(\begin{array}{cc}
\bar{a}_{+}(\bar{k}) & 0 \\
\bar{a}_{-}(\bar{k}) & 0 \\
-\bar{\Gamma}_{1}(\bar{k}) & \frac{\bar{a}_{-}(\bar{k})}{\bar{a}_{+}(\bar{k})}
\end{array}\right), & k \in \gamma \cap \mathbb{C}_{-}\end{cases}
$$

Finally, introducing

$$
M_{\text {ini }}(x, k):=\left\{\begin{array}{cc}
\check{M}(x, 0, k)\left(\begin{array}{cc}
a(k) & 0 \\
0 & \frac{1}{a(k)}
\end{array}\right), & \operatorname{Im} k>0 \\
\check{M}(x, 0, k)\left(\begin{array}{cc}
\frac{1}{\bar{a}(\bar{k})} & 0 \\
0 & \bar{a}(\bar{k})
\end{array}\right), & \operatorname{Im} k<0
\end{array}\right.
$$

the resulting jump conditions for $M_{i n i}(x, k)$ are

$$
M_{i n i,+}(x, k)=M_{i n i,-}(x, k) \mathrm{e}^{-\mathrm{i} k x \sigma_{3}} J_{i n i, 0}(k) \mathrm{e}^{\mathrm{i} k x \sigma_{3}}, \quad k \in \mathbb{R} \cup \gamma,
$$

where

$$
J_{\text {ini }, 0}(k)= \begin{cases}\left(\begin{array}{cc}
1 & \tilde{r}(k) \\
\tilde{r}(k) & 1+|\tilde{r}(k)|^{2}
\end{array}\right), & k \in \mathbb{R}, \\
\left(\begin{array}{cc}
1 & f(k) \\
0 & 1
\end{array}\right), & k \in \gamma \cap \mathbb{C}_{+}, \\
\left(\begin{array}{cc}
1 & 0 \\
-\bar{f}(\bar{k}) & 1
\end{array}\right), & k \in \gamma \cap \mathbb{C}_{-}\end{cases}
$$

with

$$
\tilde{r}(k):=\frac{b(k)}{a(k)}
$$

and

$$
f(k):=\tilde{r}_{+}(k)-\tilde{r}_{-}(k) .
$$

Now we notice that these conditions coincide with those for the spectral mapping $\left\{u_{0}(x)\right\} \mapsto$ $\{a(k), b(k)\}$ in the IV problem on the whole line with step-like initial data: $u_{0}(x)=\alpha \mathrm{e}^{-2 \mathrm{i} \beta x}$ for $x>0$ and $u_{0}(x)=0$ for $x<0$ (see [5]). This yields $u(x, 0)=u_{0}(x)=\alpha \mathrm{e}^{-2 \mathrm{i} \beta x}$ for $x>0$ due to the uniqueness of the solution of the RH problem.

Remark 3.3. In the case of the general initial data $u_{0}(x)$ (such that $u_{0}(x) \rightarrow \alpha \mathrm{e}^{-2 \mathrm{i} \beta x}$ as $x \rightarrow+\infty$ fast enough), the transformation of the original $\mathrm{RH}$ problem to a $\mathrm{RH}$ problem associated with the 
initial conditions holds as well, the difference being that in the general case, (i) the spectral function $f(k)$ is no longer related to $\tilde{r}(k)$ via (3.24) but is an independent part of the spectral data associated with $u_{0}(x)$; (ii) the residue conditions are moved to the location of possible zeros of $a(k)$.

(iii) The strategy in proving that $u(x, t)$ satisfies the Robin boundary condition follows that in the case of decaying initial data [19].

First, we notice that the RH problem (3.13) with the jump matrices defined by (3.10) for $k \in \gamma$, by (3.11) for $k \in \gamma_{1}$, and by (3.14) for $k \in \mathbb{R}$ can be considered not only for $x \geq 0$ but also for $x<0$ thus giving a continuous (at $x=0$, for all $t>0$ ) continuation of $u(x, t)(3.16)$ for $x<0$. The associated continuation of the initial data $u(x, 0)$ for $x<0$ will be discussed below; but here we observe that the structure itself of the jump matrix on $k \in \mathbb{R}$ suggests interpreting $r_{e}(k)$ as a reflection coefficient for the problem obtained on the whole line:

$$
r_{e}(k)=\frac{\bar{b}_{e}(k)}{a_{e}(k)} .
$$

In order to determine $b_{e}(k)$ and $a_{e}(k)$ correctly, we require that $a_{e}(k)$ is analytic in $\mathbb{C} \backslash\left(\gamma \cup \gamma_{1}\right)$, $a_{e}(k) \rightarrow 1$ as $k \rightarrow \infty,\left|a_{e}\right|^{-2}(k)=1+\left|r_{e}\right|^{2}(k)$ for $k \in \mathbb{R}$, and $a_{e}(k)$ has neither a zero nor a pole at $k=\mathrm{i}|q| / 2$ (cf. [7, 19]). In view of the definition (3.8) of $r_{e}(k)$, this gives

$$
a_{e}(k):= \begin{cases}a(k) \overline{a(-\bar{k})}-\frac{2 k+\mathrm{i} q}{2 k-\mathrm{i} q} b(k) \overline{b(-\bar{k})}, & \text { if } q<0, a\left(-\frac{\mathrm{i} q}{2}\right) \neq 0 \text { or } q>0, b\left(\frac{\mathrm{i} q}{2}\right)=0 \\ \frac{2 k-\mathrm{i} q}{2 k+\mathrm{i} q} a(k) \overline{a(-\bar{k})}-b(k) \overline{b(-\bar{k})}, & \text { if } q>0, b\left(\frac{\mathrm{i} q}{2}\right) \neq 0 \text { or } q<0, a\left(-\frac{\mathrm{i} q}{2}\right)=0\end{cases}
$$

and respectively

$$
b_{e}(k):= \begin{cases}b(k) a(-k)+\frac{2 k-\mathrm{i} q}{2 k+\mathrm{i} q} b(-k) a(k), & \text { if } q<0, a\left(-\frac{\mathrm{i} q}{2}\right) \neq 0 \text { or } q>0, b\left(\frac{\mathrm{i} q}{2}\right)=0 \\ \frac{2 k+\mathrm{i} q}{2 k-\mathrm{i} q} b(k) a(-k)+b(-k) a(k), & \text { if } q>0, b\left(\frac{\mathrm{i} q}{2}\right) \neq 0 \text { or } q<0, a\left(-\frac{\mathrm{i} q}{2}\right)=0\end{cases}
$$

In what follows, we will refer to these two cases of definition of $a_{e}(k)$ and $b_{e}(k)$ as Case 1 and Case 2 .

Consequently, from (3.9) we have

$$
\delta(k)=\frac{a_{e-}(k)}{a_{e+}(k)}, \quad k \in \gamma .
$$

Accordingly to the cases singled out above, let us introduce

$$
h:= \begin{cases}\frac{q}{2}, & \text { if } q<0, a\left(-\frac{\mathrm{i} q}{2}\right) \neq 0 \text { or } q>0, b\left(\frac{\mathrm{i} q}{2}\right)=0 \\ -\frac{q}{2}, & \text { if } q>0, b\left(\frac{\mathrm{i} q}{2}\right) \neq 0 \text { or } q<0, a\left(-\frac{\mathrm{i} q}{2}\right)=0\end{cases}
$$

Denote by $\hat{J}(x, t, k)$ the jump matrix in (3.13), i.e.,

$$
\hat{J}(x, t, k):=\mathrm{e}^{-\mathrm{i}\left(k x+2 k^{2} t\right) \sigma_{3}} \hat{J}_{0}(k) \mathrm{e}^{\mathrm{i}\left(k x+2 k^{2} t\right) \sigma_{3}} .
$$

Lemma 3.1. The jump matrix $\hat{J}_{0}$ in (3.13) satisfies the symmetry conditions:

$$
\begin{array}{rlrl}
\overline{\hat{J}(0, t,-k)} & =C_{-}(k) \hat{J}(0, t, k) C_{+}(k) & & \text { for } k \in \mathbb{R}, \\
(\overline{\hat{J}(0, t,-\bar{k})})^{-1} & =C_{-}(k) \hat{J}(0, t, k) C_{+}(k) \quad & \text { for } k \in \gamma \cup \gamma_{1},
\end{array}
$$


where

$$
C(k)= \begin{cases}\left(\begin{array}{cc}
a_{e}(k) & 0 \\
0 & \frac{1}{a_{e}(k)}
\end{array}\right)\left(\begin{array}{cc}
k-\mathrm{i} h & 0 \\
0 & k+\mathrm{i} h
\end{array}\right) \sigma_{1}, & \operatorname{Im} k>0, \\
\left(\begin{array}{cc}
\frac{1}{a_{e}(\bar{k})} & 0 \\
0 & a_{e}(\bar{k})
\end{array}\right)\left(\begin{array}{cc}
k-\mathrm{i} h & 0 \\
0 & k+\mathrm{i} h
\end{array}\right) \sigma_{1}, & \operatorname{Im} k<0,\end{cases}
$$

with $\sigma_{1}=\left(\begin{array}{ll}0 & 1 \\ 1 & 0\end{array}\right)$.

Proof. (i) The symmetry (3.28) follows from the symmetry of the reflection coefficient:

$$
r_{e}(-k)=r_{e}(k) \frac{a_{e}(k)}{\overline{a_{e}(k)}} \frac{k-\mathrm{i} h}{k+\mathrm{i} h} .
$$

(ii) The symmetry (3.29) can be checked directly.

Lemma 3.2. The solution $\hat{M}$ of the RH problem (3.13) satisfies the symmetry condition

$$
\overline{\hat{M}(0, t,-\bar{k})}=\sigma_{1} \bar{P}(t)\left(\begin{array}{cc}
\frac{1}{k-\mathrm{i} h} & 0 \\
0 & \frac{1}{k+\mathrm{i} h}
\end{array}\right) \bar{P}^{-1}(t) \hat{M}(0, t, k)\left(\begin{array}{cc}
k-\mathrm{i} h & 0 \\
0 & k+\mathrm{i} h
\end{array}\right) D(k) \sigma_{1},
$$

where

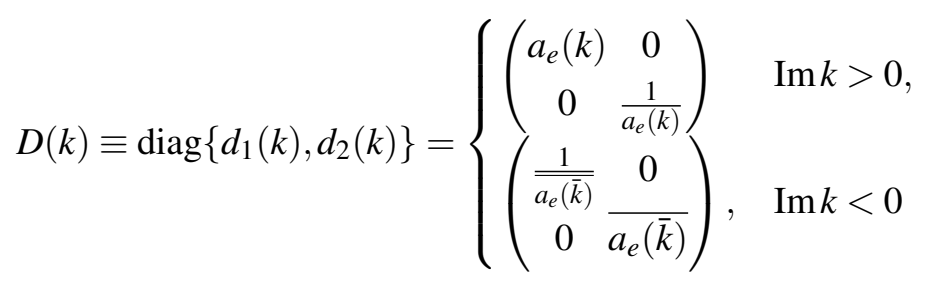

and

$$
\bar{P}(t)=\frac{1}{\Delta(t)}\left(\begin{array}{l}
\hat{M}_{11}(0, t,-\mathrm{i} h) \hat{M}_{12}(0, t, \mathrm{i} h) \\
\hat{M}_{21}(0, t,-\mathrm{i} h) \hat{M}_{22}(0, t, \mathrm{i} h)
\end{array}\right)
$$

with

$$
\begin{aligned}
\Delta(t) & =\hat{M}_{11}(0, t,-\mathrm{i} h) \hat{M}_{22}(0, t, \mathrm{i} h)-\hat{M}_{12}(0, t, \mathrm{i} h) \hat{M}_{21}(0, t,-\mathrm{i} h) \\
& =\left|\hat{M}_{11}(0, t,-\mathrm{i} h)\right|^{2}+\left|\hat{M}_{21}(0, t,-\mathrm{i} h)\right|^{2}>0 .
\end{aligned}
$$

Proof. Define $M(t, k)$ by

$$
M(t, k)=N(t, k) \overline{\hat{M}(0, t,-\bar{k})} C(k),
$$

where $N(t, k)$ is some rational (in $k$ ) function with poles outside $\mathbb{R}$. Then the symmetries (3.28) and (3.29) imply that $M(t, k)$ satisfies the jump condition $M_{+}(t, k)=M_{-}(t, k) \hat{J}(0, t, k)$. Now notice that 
the choice

$$
N(t, k)=\sigma_{1} P(t)\left(\begin{array}{cc}
\frac{1}{k-\mathrm{i} h} & 0 \\
0 & \frac{1}{k+\mathrm{i} h}
\end{array}\right) P^{-1}(t)
$$

with $P$ as in (3.34) (for the details, see [19]), implies the r.h.s. of (3.35) is nonsingular at $k= \pm \mathrm{i} h$ and approaches the identity matrix as $k \rightarrow \infty$. Then, checking the residue conditions (if any), we conclude that $M(t, k)=\hat{M}(0, t, k)$, which eventually can be written as (3.32).

Remark 3.4. The above arguments can actually prove the general symmetry formula that is valid for all $x$ and $t$ :

$$
\overline{\hat{M}(-x, t,-\bar{k})}=\sigma_{1} \bar{P}(t)\left(\begin{array}{cc}
\frac{1}{k-\mathrm{i} h} & 0 \\
0 & \frac{1}{k+\mathrm{i} h}
\end{array}\right) \bar{P}^{-1}(t) \hat{M}(x, t, k)\left(\begin{array}{cc}
k-\mathrm{i} h & 0 \\
0 & k+\mathrm{i} h
\end{array}\right) D(k) \sigma_{1} .
$$

Now we show how the symmetry relation (3.32) alone can be used to establish the Robin boundary condition for $u(x, t)$. The arguments are exactly as in the case of decaying initial data [19]; for a self-contained presentation, we repeat them here.

We first evaluate, using (3.32), the entries $\hat{M}_{11}(0, t,-\mathrm{i} h)$ and $\hat{M}_{21}(0, t,-\mathrm{i} h)$. We have:

$$
\begin{aligned}
\overline{\hat{M}_{11}(0, t,-\mathrm{i} h)} & =\lim _{k \rightarrow-\mathrm{i} h}\left(\bar{P}(t)\left(\begin{array}{cc}
\frac{1}{k-\mathrm{i} h} & 0 \\
0 & \frac{1}{k+\mathrm{i} h}
\end{array}\right) \bar{P}^{-1}(t) \hat{M}(0, t, k)\left(\begin{array}{cc}
k-\mathrm{i} h & 0 \\
0 & k+\mathrm{i} h
\end{array}\right) D(k)\right)_{22} \\
& =\bar{P}_{22}(t)\left(\bar{P}^{-1}(t) \hat{M}(0, t,-\mathrm{i} h)\right)_{22} d_{2}(-\mathrm{i} h)=\frac{1}{\Delta(t)} \hat{M}_{22}(0, t, \mathrm{i} h) d_{2}(-\mathrm{i} h) \\
& =\bar{M}_{11}(0, t,-\mathrm{i} h) \frac{d_{2}(-\mathrm{i} h)}{\Delta(t)}
\end{aligned}
$$

where we have used the basic symmetry (2.12). Similarly, we have

$$
\begin{aligned}
\overline{\hat{M}_{21}(0, t,-\mathrm{i} h)} & =\lim _{k \rightarrow-\mathrm{i} h}\left(\bar{P}(t)\left(\begin{array}{cc}
\frac{1}{k-\mathrm{i} h} & 0 \\
0 & \frac{1}{k+\mathrm{i} h}
\end{array}\right) \bar{P}^{-1}(t) \hat{M}(0, t, k)\left(\begin{array}{cc}
k-\mathrm{i} h & 0 \\
0 & k+\mathrm{i} h
\end{array}\right) D(k)\right)_{12} \\
& =\bar{P}_{12}(t)\left(\bar{P}^{-1}(t) \hat{M}(0, t,-\mathrm{i} h)\right)_{22} d_{2}(-\mathrm{i} h)=\frac{1}{\Delta(t)} \hat{M}_{12}(0, t, \mathrm{i} h) d_{2}(-\mathrm{i} h) \\
& =-\hat{M}_{21}(0, t,-\mathrm{i} h) \frac{d_{2}(-\mathrm{i} h)}{\Delta(t)} .
\end{aligned}
$$

Comparing (3.37) and (3.38) gives

$$
\overline{\hat{M}_{11}(0, t,-\mathrm{i} h)}(1-\theta)=0 \text { and } \overline{\hat{M}_{21}(0, t,-\mathrm{i} h)}(1+\theta)=0,
$$

where

$$
\theta=\frac{d_{2}(-\mathrm{i} h)}{\Delta}
$$

which implies that either $\hat{M}_{11}(0, t,-\mathrm{i} h)=0$ or $\hat{M}_{21}(0, t,-\mathrm{i} h)=0$ for all $t \geq 0$. 
Now recall that $\Psi(t, k):=\left(\hat{M}_{11}(0, t, k) \mathrm{e}^{-2 \mathrm{i} k^{2} t}, \hat{M}_{21}(0, t, k) \mathrm{e}^{-2 \mathrm{i} k^{2} t}\right)^{T}$ satisfies the differential equation (2.4) with $u=u(0, t)$ and $u_{x}=u_{x}(0, t)$, i.e.

$$
\begin{aligned}
& \frac{d \Psi_{1}}{d t}+2 \mathrm{i} k^{2} \Psi_{1}=\mathrm{i}|u(0, t)|^{2} \Psi_{1}+\left(2 k u(0, t)+\mathrm{i} u_{x}(0, t)\right) \Psi_{2}, \\
& \frac{d \Psi_{2}}{d t}-2 \mathrm{i} k^{2} \Psi_{2}=-\mathrm{i}|u(0, t)|^{2} \Psi_{2}+\left(-2 k \bar{u}(0, t)+\mathrm{i} \bar{u}_{x}(0, t)\right) \Psi_{1} .
\end{aligned}
$$

From (3.41) it follows that if $\Psi_{1}(t,-\mathrm{i} h)=0$ for all $t \geq 0$ then $-2 \mathrm{i} h u(0, t)+\mathrm{i} u_{x}(0, t) \equiv 0$, or $u_{x}(0, t)-$ $2 h u(0, t) \equiv 0$; if $\Psi_{2}(t,-\mathrm{i} h)=0$ then $2 \mathrm{i} h \bar{u}(0, t)+\mathrm{i} \bar{u}_{x}(0, t) \equiv 0$, or $u_{x}(0, t)+2 h u(0, t) \equiv 0$. Observe that, according to (3.27), $h$ can be either $q / 2$ or $-q / 2$. But since the initial data satisfy the boundary condition $u_{x}(0,0)+q u(0,0)=0$, by continuity it follows that this condition holds for all $t$.

A closer look at (3.39) and (3.40) reveals that one can specify precisely whether (a) $\hat{M}_{11}(0, t,-\mathrm{i} h)=0$ or (b) $\hat{M}_{21}(0, t,-\mathrm{i} h)=0$ occurs, depending on the sign of $q$ and the properties of $a(\mathrm{i}|q| / 2)$ and $b(\mathrm{i}|q| / 2)$. Indeed, since $\Delta=\left|\hat{M}_{11}(-\mathrm{i} h)\right|^{2}+\left|\hat{M}_{21}(-\mathrm{i} h)\right|^{2}>0$, the choice between (a) and (b) is determined by the sign of $d_{2}(-\mathrm{i} h)$. According to (3.25) and (3.27), one can distinguish four cases.

(i) If $q>0$ and $b(\mathrm{i} q / 2)=0$, then $h=\frac{q}{2}>0$ and thus (see (3.33)) $d_{2}(-\mathrm{i} h)=\overline{a_{e}\left(\frac{\mathrm{i} q}{2}\right)}$. In turn, from (3.25) it follows that in this case, $\overline{a_{e}\left(\frac{\mathrm{i} q}{2}\right)}=\left|a\left(\frac{\mathrm{i} q}{2}\right)\right|^{2}>0$ and thus $1+\frac{d_{2}(-\mathrm{i} h)}{\Delta}>0$, which implies (see(3.39)) that $\hat{M}_{21}(0, t,-\mathrm{i} h)=0$.

(ii) If $q<0$ and $a(-\mathrm{i} q / 2) \neq 0$, then $h=\frac{q}{2}<0$ and thus $d_{2}(-\mathrm{i} h)=\left(a_{e}\left(-\frac{\mathrm{i} q}{2}\right)\right)^{-1}=\left|a\left(-\frac{\mathrm{i} q}{2}\right)\right|^{-2}>$ 0 . Hence, in this case one also has $1+\frac{d_{2}(-\mathrm{i} h)}{\Delta}>0$ and thus $\hat{M}_{21}(0, t,-\mathrm{i} h)=0$.

(iii) If $q<0$ and $a(-\mathrm{i} q / 2)=0$, then $h=-\frac{q}{2}>0$ and thus $d_{2}(-\mathrm{i} h)=\overline{a_{e}\left(-\frac{\mathrm{i} q}{2}\right)}=-\left|b\left(-\frac{\mathrm{i} q}{2}\right)\right|^{2}<0$. Hence, in this case $1-\frac{d_{2}(-\mathrm{i} h)}{\Delta}>0$, which implies that $\hat{M}_{11}(0, t,-\mathrm{i} h)=0$.

(iv) If $q>0$ and $b(\mathrm{i} q / 2) \neq 0$, then $h=-\frac{q}{2}<0$ and thus $d_{2}(-\mathrm{i} h)=\left(a_{e}\left(\frac{\mathrm{i} q}{2}\right)\right)^{-1}=-\left|b\left(\frac{\mathrm{i} q}{2}\right)\right|^{-2}<$ 0 . Hence, in this case one also has $1-\frac{d_{2}(-\mathrm{i} h)}{\Delta}>0$, which implies that $\hat{M}_{11}(0, t,-\mathrm{i} h)=0$.

Summarizing, we see that $\hat{M}_{21}(0, t,-\mathrm{i} h)=0$ corresponds to $h=\frac{q}{2}$ while $\hat{M}_{11}(0, t,-\mathrm{i} h)=0$ corresponds to $h=-\frac{q}{2}$, which is indeed consistent with the fact that (3.41) implies $u_{x}(0, t)+q u(0, t)=$ 0 for all $t$.

\section{Initial value problem associated with the initial boundary value problem (1.2)}

Theorem 4.1. Let $\hat{M}(x, t, k)$ be the solution of the RH problem with the jump matrices defined in terms of $q$ and $a(k)$ and $b(k)$ from (2.15) via (3.8)-(3.14). Let $u(x, t)$ be determined by $\hat{M}(x, t, k)$ via (3.16). Then:

- $u(x, t)$ satisfies the NLS equation for $-\infty<x<\infty, t>0$;

- $u(x, 0)=\alpha \mathrm{e}^{-2 \mathrm{i} \beta x}$ for $x>0$;

- $u(x, 0)=\alpha \mathrm{e}^{\mathrm{i} \phi} \mathrm{e}^{2 \mathrm{i} \beta x}$ for $x<0$,

where

$$
\phi=\frac{-i}{\pi} \int_{\gamma_{1}} \frac{\log (2 \xi-\mathrm{i}|q|)-\log (2 \xi+\mathrm{i}|q|)}{\left(\sqrt{(\xi+\beta)^{2}+\alpha^{2}}\right)_{+}} \mathrm{d} \xi .
$$


Moreover,

$$
u_{x}(0, t)+q u(0, t)=0 \quad \text { for } t>0 .
$$

Remark 4.1. For $t>0, u(x, t)$ decays to $u^{p}(x, t)$ as $x \rightarrow+\infty$ and to $\tilde{u}^{p}(x, t):=\alpha \mathrm{e}^{2 \mathrm{i} \beta x+2 \mathrm{i} \omega t+\mathrm{i} \phi}$ as $x \rightarrow-\infty$; but the rate of this decay can be slow, which is due to the slow decay of $r_{e}(k)$ as $\frac{1}{k}$ as $k \rightarrow \infty$. In turn, the latter is due to a discontinuity of $u(x, 0)$ at $x=0$. Accordingly, the uniqueness for the initial value problem is a subtle issue which requires an additional investigation.

Proof. In view of Theorem 3.1, the only item to be proved is the formula $u(x, 0)=\alpha \mathrm{e}^{\mathrm{i} \phi} \mathrm{e}^{2 \mathrm{i} \beta x}$ giving the continuation of the initial data to the left half-line. We will prove it via a series of transformations of the original RH problem in the spirit of the nonlinear steepest descent method [8].

First, let us express the jump matrix $\hat{J}_{0}(k)$ in (3.13) in terms of $a_{e}(k)$ and $b_{e}(k)$ determined by (3.25) and (3.26).

Lemma 4.1. The off-diagonal entries in (3.11), $k \in \gamma_{1}$, can be written as follows:

$$
\tilde{\Gamma}_{+}(k)-\tilde{\Gamma}_{-}(k)=\left\{\begin{array}{ll}
\frac{2 k+\mathrm{i} q}{2 k-\mathrm{i} q} \frac{\mathrm{i}}{a_{e+1}(k) a_{e-}(k)}, & \text { case } 1 \\
\frac{2 k-\mathrm{i} q}{2 k+\mathrm{i} q} \frac{\mathrm{i}}{a_{e+}(k) a_{e-}(k)}, & \text { case } 2
\end{array} .\right.
$$

The proof follows the direct calculation using the definition of $\tilde{\Gamma}$ and the identity (2.14) from Lemma 2.1.

Thus $\hat{J}_{0}(k)$ can be written as follows:

$$
\hat{J}_{0}(k)= \begin{cases}\left(\begin{array}{cc}
\frac{a_{e-}(k)}{a_{e+}(k)} & \mathrm{i} \\
0 & \frac{a_{e+}(k)}{a_{e-}(k)}
\end{array}\right), & k \in \gamma \cap \mathbb{C}_{+} \\
\left(\begin{array}{cc}
1 & 0 \\
\frac{2 k \pm \mathrm{i} q}{2 k \mp \mathrm{i} q} \frac{\mathrm{i}}{a_{e+1}(k) a_{e-}(k)} & 1
\end{array}\right), & k \in \gamma_{1} \cap \mathbb{C}_{+} \\
\left(\begin{array}{cc}
1 & \bar{r}_{e}(k) \\
0 & 1
\end{array}\right)\left(\begin{array}{cc}
1 & 0 \\
r_{e}(k) & 1
\end{array}\right), & k \in \mathbb{R}\end{cases}
$$

where $r_{e}=\frac{\bar{b}_{e}}{a_{e}}$, and

$$
\hat{J}_{0}(k)=\left(\begin{array}{cc}
0 & 1 \\
-1 & 0
\end{array}\right) \overline{\hat{J}_{0}(\bar{k})}\left(\begin{array}{cc}
0 & -1 \\
1 & 0
\end{array}\right)
$$

for the parts of the contour in $\mathbb{C}_{-}$, i.e., for $k \in\left(\gamma \cup \gamma_{1}\right) \cap \mathbb{C}_{-}$.

Now define $M^{(1)}$ by

$$
M^{(1)}(x, t, k)=\left\{\begin{array}{cc}
\hat{M}(x, t, k)\left(\begin{array}{cc}
a_{e}(k) & 0 \\
0 & a_{e}^{-1}(k)
\end{array}\right), & k \in \mathbb{C}_{+} \\
\hat{M}(x, t, k)\left(\begin{array}{cc}
\overline{a_{e}^{-1}(\bar{k})} & 0 \\
0 & \overline{a_{e}(\bar{k})}
\end{array}\right), & k \in \mathbb{C}_{-}
\end{array}\right.
$$

Then the jump condition for $M^{(1)}(x, 0, k)$ is as follows:

$$
M_{+}^{(1)}(x, 0, k)=M_{-}^{(1)}(x, 0, k) J^{(1)}(x, 0, k),
$$


where

$$
J^{(1)}(x, 0, k)= \begin{cases}\left(\begin{array}{ll}
1 \frac{\mathrm{ie}-2 i k x}{a_{e-}(k) a_{e+}(k)} \\
0 & 1
\end{array}\right), & k \in \gamma \cap \mathbb{C}_{+} \\
\left(\begin{array}{cc}
\frac{a_{e+}(k)}{a_{e-}(k)} & 0 \\
\frac{2 k \pm \mathrm{i} q}{2 k \mp \mathrm{i} q} \mathrm{ie}^{2 \mathrm{i} k x} \frac{a_{e-}(k)}{a_{e+}(k)}
\end{array}\right), & k \in \gamma_{1} \cap \mathbb{C}_{+} \\
\left(\begin{array}{cr}
1 & 0 \\
\tilde{r}_{e}(k) \mathrm{e}^{2 \mathrm{i} k x} & 1
\end{array}\right)\left(\begin{array}{cc}
1 & \tilde{r}_{e}(k) \mathrm{e}^{-2 \mathrm{i} k x} \\
0 & 1
\end{array}\right), & k \in \mathbb{R}\end{cases}
$$

where $\tilde{r}_{e}=\frac{b_{e}}{a_{e}}$, and

$$
\boldsymbol{J}^{(1)}(x, 0, k)=\left(\begin{array}{cc}
0 & 1 \\
-1 & 0
\end{array}\right) \overline{\boldsymbol{J}^{(1)}(x, 0, \bar{k})}\left(\begin{array}{cc}
0 & -1 \\
1 & 0
\end{array}\right), \quad k \in\left(\gamma \cup \gamma_{1}\right) \cap \mathbb{C}_{-} .
$$

The next transformation aims at removing the growing (as $x \rightarrow-\infty$ ) exponential in the jump matrix on $\gamma_{1}$ (the " $g$-function" step, by now standard in the method of nonlinear steepest descent, see, e.g., $[5,6])$. Define $M^{(2)}$ by

$$
M^{(2)}(x, 0, k)=\mathrm{e}^{-\mathrm{i} \beta x \sigma_{3}} M^{(1)}(x, 0, k) \mathrm{e}^{(-\mathrm{i} k x+\mathrm{i} \hat{X}(k) x) \sigma_{3}},
$$

where (cf. (2.8)) $\hat{X}(k)=\sqrt{(k+\beta)^{2}+\alpha^{2}}$ with the branch cut $\gamma_{1}$ and $\hat{X}(k)=k+\beta=o(1)$ as $k \rightarrow \infty$ (so that the large- $k$ behavior is preserved: $M^{(2)} \rightarrow E$ as $k \rightarrow \infty$ ). Then $\hat{X}_{+}+\hat{X}_{-}=0$ for $k \in \gamma_{1}$, and the jump matrix $J^{(2)}$ in the jump relation $M_{+}^{(2)}(x, 0, k)=M_{-}^{(2)}(x, 0, k) J^{(2)}(x, 0, k)$ takes the form

$$
J^{(2)}(x, 0, k)= \begin{cases}\left(\begin{array}{ll}
1 & \frac{\mathrm{ie}-2 \mathrm{i} \hat{X}(k) x}{a_{e-}(k) a_{e+}(k)} \\
0 & 1
\end{array}\right), & k \in \gamma \cap \mathbb{C}_{+} \\
\left(\begin{array}{cc}
\frac{a_{e+}(k)}{a_{e-}(k)} \mathrm{e}^{\mathrm{i}\left(\hat{X}_{+}(k)-\hat{X}_{-}(k)\right) x} & 0 \\
\frac{2 k \pm \mathrm{i} q}{2 k \neq \mathrm{i} q} & \frac{a_{e-}(k)}{a_{e+}(k)} \mathrm{e}^{-\mathrm{i}\left(\hat{X}_{+}(k)-\hat{X}_{-}(k)\right) x}
\end{array}\right), & k \in \gamma_{1} \cap \mathbb{C}_{+} \\
\left(\begin{array}{cr}
1 & 0 \\
\tilde{\tilde{r}}_{e}(k) \mathrm{e}^{2 \mathrm{i} \hat{X}(k) x} & 1
\end{array}\right)\left(\begin{array}{cc}
1 & \tilde{r}_{e}(k) \mathrm{e}^{-2 \mathrm{i} \hat{X}(k) x} \\
0 & 1
\end{array}\right), & k \in \mathbb{R}\end{cases}
$$

and

$$
J^{(2)}(x, 0, k)=\left(\begin{array}{cc}
0 & 1 \\
-1 & 0
\end{array}\right) \overline{\boldsymbol{J}^{(2)}(x, 0, \bar{k})}\left(\begin{array}{cc}
0 & -1 \\
1 & 0
\end{array}\right), \quad k \in\left(\gamma \cup \gamma_{1}\right) \cap \mathbb{C}_{-} .
$$

Finally, introduce $M^{(3)}$ by

$$
M^{(3)}(x, 0, k)=\left\{\begin{array}{cc}
\left(\begin{array}{cc}
f(\infty) & 0 \\
0 & f^{-1}(\infty)
\end{array}\right) M^{(2)}(x, 0, k)\left(\begin{array}{cc}
f^{-1}(k)-f(k) h(k) \mathrm{e}^{-2 \mathrm{i} \hat{X}(k) x} \\
0 & f(k)
\end{array}\right), & k \in \mathbb{C}_{+} \\
\left(\begin{array}{cc}
\bar{f}^{-1}(\infty) & 0 \\
0 & \bar{f}(\infty)
\end{array}\right) M^{(2)}(x, 0, k)\left(\begin{array}{cc}
\bar{f}(\bar{k}) & 0 \\
\bar{f}(\bar{k}) \bar{h}(\bar{k}) \mathrm{e}^{2 \mathrm{i} \overline{\mathrm{X}}(\bar{k}) x} & \bar{f}^{-1}(\bar{k})
\end{array}\right), & k \in \mathbb{C}_{-}
\end{array}\right.
$$


where the scalar functions $h(k)$ and $f(k)$ are to be determined. Assuming that $h(k)$ and $f(k)$ are analytic for $k \in \mathbb{C} \backslash\left\{\gamma \cup \gamma_{1}\right\}$ and that $\bar{f}(\bar{k})=f^{-1}(k)$, the jump matrix $J^{(3)}$ in $M_{+}^{(3)}(x, 0, k)=$ $M_{-}^{(3)}(x, 0, k) J^{(3)}(x, 0, k)$ takes the form:

- for $k \in \gamma_{1} \cap \mathbb{C}_{+}$:

$$
J^{(3)}(x, 0, k)=\left(\begin{array}{cc}
f_{+}^{-1} f_{-}\left(\frac{a_{e+}}{a_{e-}}+h_{-} \frac{2 k \pm \mathrm{i} q}{2 k \mp \mathrm{i} q}\right) \mathrm{e}^{-2 \mathrm{i} \hat{X}_{-} x} & f_{-} f_{+}\left(-h_{+}\left(\frac{a_{e+}}{a_{e-}}+h_{-} \frac{2 k \pm \mathrm{i} q}{2 k \mp \mathrm{i} i} \mathrm{i}\right)+h_{-} \frac{a_{e-}}{a_{e+}}\right) \\
f_{+}^{-1} f_{-}^{-1} \frac{2 k \pm \mathrm{i} q}{2 k \mp \mathrm{i} q} & f_{-}^{-1} f_{+}\left(\frac{a_{e-}}{a_{e+}}-h_{+} \frac{2 k \pm \mathrm{i} q}{2 k \mp \mathrm{i} i}\right) \mathrm{e}^{-2 \mathrm{i} \hat{X}_{+}}
\end{array}\right)
$$

- for $k \in \gamma \cap \mathbb{C}_{+}$:

$$
J^{(3)}(x, 0, k)=\left(\begin{array}{l}
1\left(\frac{\mathrm{i}}{a_{e+}(k) a_{e-}(k)}+h_{+}(k)-h_{-}(k)\right) f_{-}(k) f_{+}(k) \mathrm{e}^{-2 \mathrm{i} \hat{X}(k) x} \\
1
\end{array}\right)
$$

- for $k \in \gamma \cap \mathbb{C}_{-}$and $k \in \gamma_{1} \cap \mathbb{C}_{-}: \hat{J}^{(3)}(k)=\left(\begin{array}{cc}0 & 1 \\ -1 & 0\end{array}\right) \overline{\hat{J}^{(3)}(\bar{k})}\left(\begin{array}{cc}0 & -1 \\ 1 & 0\end{array}\right)$

- for $k \in \mathbb{R}$ :

$$
J^{(3)}(x, 0, k)=\left(\begin{array}{cc}
1 & 0 \\
\left(\overline{\tilde{r}}_{e}(k)-\bar{h}(k)\right) f^{-2}(k) \mathrm{e}^{2 \mathrm{i} \hat{X}(k) x} & 1
\end{array}\right)\left(\begin{array}{c}
1\left(\tilde{r}_{e}(k)-h(k)\right) f^{2}(k) \mathrm{e}^{-2 \mathrm{i} \hat{X}(k) x} \\
0
\end{array}\right)
$$

Lemma 4.2. Setting $h(k):=\tilde{r}_{e}(k)$, the jump matrix $J^{(3)}(x, 0, k)$ simplifies to

$$
J^{(3)}(x, 0, k)=\left(\begin{array}{cc}
0 & f_{-}(k) f_{+}(k) \frac{2 k \mp \mathrm{i} q}{2 k \pm \mathrm{i} q} \\
f_{+}^{-1}(k) f_{-}^{-1}(k) \frac{2 k \pm \mathrm{i} q}{2 k \mp \mathrm{i} q} \mathrm{i} & 0
\end{array}\right), \quad k \in \gamma_{1}
$$

(so that $J^{(3)} \equiv E$ for $k \in \gamma$ and for $\left.k \in \mathbb{R}\right)$. Moreover, $M^{(3)}(x, 0, k)$ is a piecewise analytic function (having no poles).

Indeed, in the case of $a(k)$ and $b(k)$ given by (2.15), $b_{e}(k)$ is analytic (similarly to $a_{e}(k)$ ) in $k \in \mathbb{C} \backslash\left\{\gamma \cup \gamma_{1}\right\}$; moreover, direct calculations give

$$
\frac{b_{e+}(k)}{a_{e+}(k)}=-\frac{a_{e-}(k)}{a_{e+}(k)} \frac{2 k \mp \mathrm{i} q}{2 k \pm \mathrm{i} q} \mathrm{i}, \quad \frac{b_{e-}(k)}{a_{e-}(k)}=\frac{a_{e+}(k)}{a_{e-}(k)} \frac{2 k \mp \mathrm{i} q}{2 k \pm \mathrm{i} q} \mathrm{i}
$$

and thus $J^{(3)}$ takes the required form for $k \in \gamma_{1} \cap \mathbb{C}_{+}$. On the other hand, for $k \in \gamma$ one has

$$
\frac{b_{e-}(k)}{a_{e-}(k)}-\frac{b_{e+}(k)}{a_{e+}(k)}=\frac{\mathrm{i}}{a_{e+}(k) a_{e-}(k)}
$$

and thus $J^{(3)}$ reduces to $E$ on $k \in \gamma \cap \mathbb{C}_{+}$.

The fact that $M^{(3)}(x, 0, k)$ has no poles at the zeros of $a_{e}(k)$ (if any) can be checked directly.

Now let us specify $f(k)$ as a solution of the following factorization problem:

- $f(k)$ is analytic in $\mathbb{C} \backslash \gamma_{1}$

- $f(k)$ is bounded as $k \rightarrow \infty$

- the limiting values $f_{ \pm}$of $f$ on $\gamma_{1}$ are related as follows: $f_{+}(k) f_{-}(k)=\frac{2 k \pm \mathrm{i} q}{2 k \mp \mathrm{i} q}$. 
Noting that $\tilde{f}:=\frac{\log f}{\hat{X}}$ has to satisfy the jump condition $\tilde{f}_{+}-\tilde{f}_{-}=\frac{\log \eta}{\tilde{X}_{+}}$and $\tilde{f} \rightarrow 0$ as $k \rightarrow \infty$, the Cauchy-type integral formula for $f$ follows:

$$
f(k)=\exp \left\{\frac{\hat{X}(k)}{2 \pi} \int_{\gamma_{1}} \frac{\log \eta(\xi)}{\hat{X}_{+}(\xi)(\xi-k)} d \xi\right\},
$$

where $\eta(k):=\frac{2 k \pm \mathrm{i} q}{2 k \mp i q}$. Particularly, we have

$$
f(\infty)=\exp \left\{-\frac{1}{2 \pi} \int_{\gamma_{1}} \frac{\log \eta(\xi)}{\hat{X}_{+}(\xi)} d \xi\right\} .
$$

With $f$ determined in this way, $J^{(3)}(x, 0, k)$ reduces to a matrix with constant entries and thus the RH problem for $M^{(3)}$ reduces to

$$
M_{+}^{(3)}(x, 0, k)=M_{-}^{(3)}(x, 0, k)\left(\begin{array}{cc}
0 & \mathrm{i} \\
\mathrm{i} & 0
\end{array}\right), \quad k \in \gamma_{1}
$$

- $M^{(3)}(x, 0, k) \rightarrow E$ as $k \rightarrow \infty$

The unique solution of this RH problem, having singularities at the end points of $\gamma_{1}$ of order not greater than $1 / 2$, is given explicitly:

$$
M_{+}^{(3)}(x, 0, k)=\frac{1}{2}\left(\begin{array}{l}
\varkappa(k)+\varkappa(k)^{-1} \varkappa(k)-\varkappa(k)^{-1} \\
\varkappa(k)-\varkappa(k)^{-1} \varkappa(k)+\varkappa(k)^{-1}
\end{array}\right)
$$

with $\varkappa(k)=\left(\frac{k+\beta-\mathrm{i} \alpha}{k+\beta+\mathrm{i} \alpha}\right)^{\frac{1}{4}}$.

Going back to $\hat{M}$ we have for $\operatorname{Im} k>0$ :

$$
\begin{aligned}
\hat{M}(x, 0, k)= & \mathrm{e}^{\mathrm{i} \beta x \sigma_{3}}\left(\begin{array}{cc}
f^{-1}(\infty) & 0 \\
0 & f(\infty)
\end{array}\right) M^{(3)}(x, 0, k)\left(\begin{array}{cc}
f(k) & f(k) h(k) \mathrm{e}^{-2 \mathrm{i} \hat{X}(k) x} \\
0 & f^{-1}(k)
\end{array}\right) \\
& \times \mathrm{e}^{(\mathrm{i} k x-\mathrm{i} \hat{X}(k) x) \sigma_{3}}\left(\begin{array}{cc}
a_{e}^{-1}(k) & 0 \\
0 & a_{e}(k)
\end{array}\right)
\end{aligned}
$$

Taking into account that $\varkappa(k)=1+\frac{\alpha}{2 \mathrm{i} k}+O\left(\frac{1}{k^{2}}\right)$ as $k \rightarrow \infty$ we conclude that for all $x<0$,

$$
u(x, 0)=\lim _{k \rightarrow \infty} 2 \mathrm{i} k \hat{M}_{12}(x, 0, k)=\alpha \mathrm{e}^{2 \mathrm{i} \beta x} f^{-2}(\infty) .
$$

Finally we notice that in the case of exact exponential initial data, $u_{0}(x)=\alpha \mathrm{e}^{-2 \mathrm{i} \beta x}$, case 1 is realized with $q<0$ whereas case 2 is realized with $q>0$ and thus the additional phase factor, coming from $f^{-2}(\infty)$, see (4.13), takes the form as in (4.1).

Remark 4.2. Similarly to Theorem 3.1, a more general result holds: the RH problem constructed from the spectral functions determined by $u(x, 0)=u_{0}(x), x>0$ with $u_{0}(x) \rightarrow \alpha \mathrm{e}^{-2 \mathrm{i} \beta x}$ as $x \rightarrow$ $+\infty$ gives rise to $u(x, t)$ such that $u(x, 0) \rightarrow \alpha \mathrm{e}^{\mathrm{i} \phi} \mathrm{e}^{2 \mathrm{i} \beta x}$ as $x \rightarrow-\infty$. Similarly to the case of exact exponential initial data, the proof is based on a sequence of transformations of the RH problem leading the the same model problem with the jump (4.14). 
A major difference, comparing to the case of exact exponential initial data, is that in passing from $M^{(2)}$ to $M^{(3)}$, one cannot expect to determine $h(k)$ as an analytic function in the whole halfplane $\mathbb{C}_{+}$that allows getting rid of the diagonal terms in the jump matrix on $\gamma_{1}$, see (4.10), and simultaneously, reducing (4.11) and (4.12) exactly to the identity matrix. Instead, lens-shaped domains are to be introduced containing $\gamma_{1} \cap \mathbb{C}_{+}, \gamma_{1} \cap \mathbb{C}_{-}$and the rays $(-\infty,-\beta)$ and $(-\beta, \infty)$, together with appropriated functions, analytic in the lenses, that approximate respectively $-\frac{a_{e}-(k)}{a_{e+}(k)} \frac{2 k \mp \mathrm{i} q}{2 k \pm \mathrm{i} q} \mathrm{i}$, $\frac{a_{e+}(k)}{a_{e-}(k)} \frac{2 k \neq \mathrm{i} q}{2 k \pm \mathrm{i} q} \mathrm{i}$ on $\gamma_{1} \cap \mathbb{C}_{+}$and $\frac{b_{e}(k)}{a_{e}(k)}$ on $\mathbb{R}$. Then $M^{(3)}$ is determined by $M^{(2)}$ multiplied, in each lens, by a particular triangular factor similar to (4.10)-(4.12), which leads to the jump matrix $J^{(3)}$ involving, apart from (4.14) for $k \in \gamma_{1}$, the jump matrices on the boundaries of the lenses that approach the identity matrix as $x \rightarrow-\infty$. As a result, $u(x, 0)$ obtained via the solution of the $\mathrm{RH}$ problem does not coincide, for all $x<0$, with $\alpha \mathrm{e}^{2 \mathrm{i} \beta x}$ as in Theorem 4.1 but approaches this exponential as $x \rightarrow-\infty$. The opening of lenses idea is also standard in the method of steepest descent; see [5,6] in the context of focusing NLS with steplike initial data.

\section{Large-time asymptotics: rarefaction case $(\beta<0)$}

The RH problems in the cases $\beta>0$ and $\beta<0$ differ only in the positions of $\gamma$ and $\gamma_{1}$. This difference, being irrelevant in proving that the solution of the $\mathrm{RH}$ problem indeed satisfies the Robin boundary condition and the prescribed initial condition, becomes important in the analysis of the large-time behavior of the solution of the IBV problem (1.2) and the associated initial value problem (see theorem 4.1).

In the case $\beta>0, \gamma$ is located in the right half-plane and $\gamma_{1}$ is located in the left half-plane. The asymptotic analysis, based on the Deift-Zhou nonlinear steepest descent method [8], of the RH problem in this situation is similar to that with $q=0$, which was done in [6]. Namely, in [6] it was shown that a shock-type large-time behavior occurs: one can specify $\xi_{j}=\xi_{j}(\alpha, \beta), j=1,2$ such that (i) in the sector $\left|\frac{x}{t}\right|<\xi_{1}$ of the $(x, t)$ plane (containing, in particular, the vertical rays corresponding to fixed $x$ and $t \rightarrow+\infty$ ), the large-time behavior of the solution of the initial value problem is oscillatory and can be described in terms of the single phase theta functions; (ii) in the sectors $\left|\frac{x}{t}\right|>\xi_{2}$, the asymptotics has a plane wave form; (iii) in two transition sectors $\xi_{1}<\left|\frac{x}{t}\right|<\xi_{2}$, the asymptotics is expressed in terms of the two phase theta functions. Following [6], one can show that the presence of $q \neq 0$ does not change the location of the boundaries of the sectors and the qualitative behavior of the solution in the corresponding sectors, but it does break the symmetry for the solution of the IV problem with respect to the $t$-axis, by adding a factor (similar to $\mathrm{e}^{\mathrm{i} \phi}$ in Theorem 4.1) to the corresponding theta-function asymptotic formulas.

In the case $\beta<0, \gamma$ and $\gamma_{1}$ interchange their locations, which implies, in particular, that in the sector $|x / t| \leq 4|\beta|$ (particularly, for all fixed $x$ ), the exponential factors in the jump matrices on $\gamma$ and $\gamma_{1}$, see (3.10), (3.11), (3.13), are all decaying, as $t \rightarrow \infty$. Consequently, in this sector, the contribution of the jumps across $\gamma$ and $\gamma_{1}$ is vanishing as $t \rightarrow \infty$ and thus $u(x, t)$ decays to 0 unless there are $k_{j}, j=1,2, \ldots$ such that $\left|\operatorname{Re} k_{j}\right|<|\beta|$ and $a_{e}\left(k_{j}\right)=0$, which generate residue conditions for the RH problem.

Specifically, consider the case of pure exponential initial conditions: $u(x, 0)=\alpha \mathrm{e}^{-2 \mathrm{i} \beta x}$ for $x>0$. Depending of the sign of $q$, we have two situations:

- if $q<0$, then $a_{e}(k)$ has no zeros with $|\operatorname{Re} k|<|\beta|$. 
- if $q>0$, then $a_{e}(k)$ has exactly one zero, which is located on the imaginary axis: $a_{e}(\mathrm{i} \zeta)=0$ with some $\zeta>0$.

Consequently, if $q<0$, then $u(x, t)$ is rapidly decaying as $t \rightarrow \infty$ in the sector $\left|\frac{x}{t}\right|<4|\beta|$. As for the case $q>0$, in this sector $u(x, t)$ behaves like a breather. In order to demonstrate this, we first notice that the residue conditions (3.17) can be written in terms of $a_{e}(k)$ and $b_{e}(k)$ only. Indeed, in the case of pure exponential initial data, $b_{e}(k)$ is analytic in $\mathbb{C} \backslash\left(\gamma \cup \gamma_{1}\right)$ and, using the symmetries in (2.15), can be written as (cf. (3.26))

$$
b_{e}(k):= \begin{cases}b(k) \overline{a(-\bar{k})}-\frac{2 k-\mathrm{i} q}{2 k+\mathrm{i} q} \overline{b(-\bar{k})} a(k), & \text { if } q<0, \\ \frac{2 k+\mathrm{i} q}{2 k-\mathrm{i} q} b(k) \overline{a(-\bar{k})}-\overline{b(-\bar{k})} a(k), & \text { if } q>0 .\end{cases}
$$

Then, using the definition (3.25) of $a_{e}(k)$, it is readily seen that if $k^{*}$ is such that $a_{e}\left(k^{*}\right)=0$, then (cf. (3.17))

$$
\frac{1}{b_{e}\left(k^{*}\right)}=\overline{\frac{b\left(-\bar{k}^{*}\right)}{a\left(k^{*}\right)}} \chi\left(k^{*}\right)
$$

with $\chi(k)$ defined in (3.18), and thus the residue conditions can be written, in both cases, as

$$
\begin{aligned}
& \operatorname{Res}_{k_{j}} \hat{M}^{(1)}(x, t, k)=\frac{\mathrm{e}^{2 \mathrm{i} \Theta\left(x, t, k_{j}\right)}}{\dot{a}_{e}\left(k_{j}\right) b_{e}\left(k_{j}\right)} \hat{M}^{(2)}\left(x, t, k_{j}\right), \\
& \operatorname{Res}_{\bar{k}_{j}} \hat{M}^{(2)}(x, t, k)=\frac{\mathrm{e}^{-2 \mathrm{i} \Theta\left(x, t, k_{j}\right)}}{\overline{\dot{a}}_{e}\left(k_{j}\right) b_{e}\left(k_{j}\right)} \hat{M}^{(1)}\left(x, t, \bar{k}_{j}\right) .
\end{aligned}
$$

Then, applying the procedure of reducing a (singular) RH problem with residue conditions to a regular RH problem (see, e.g., [9]), one arrives at the following theorem.

Theorem 5.1. Consider the initial boundary value problem (1.2), where $u_{0}(x)=\alpha \mathrm{e}^{-2 \mathrm{i} \beta x}$ with $\beta<0$ and $q>0$. Then for $0 \leq \frac{x}{t}<4|\beta|$, the solution $u(x, t)$ has the asymptotics

$$
u(x, t)=\tilde{u}(x, t)(1+o(1)), \quad t \rightarrow \infty,
$$

where

$$
\tilde{u}(x, t)=\mathrm{ie}^{-\mathrm{i} \eta} \frac{\sqrt{2 \omega}}{\cosh \left(\sqrt{2 \omega} x+\phi_{0}\right)} \mathrm{e}^{2 \mathrm{i} \omega t}
$$

Here the parameters are defined as follows:

- $\omega=2 \zeta^{2}$, where $\zeta>0$ is the (unique) solution of the equation $a_{e}(\mathrm{i} \zeta)=0$, and $a_{e}(k)$ is determined in (3.25) with $a(k)$ and $b(k)$ determined by (2.15) and (2.8);

- $\eta=\arg (\gamma)$, where $\gamma=\frac{1}{a_{e}(\mathrm{i} \zeta) b_{e}(\mathrm{i} \zeta)}$;

- $\phi_{0}=\log \frac{2 \zeta}{|\gamma|}$. 
In particular, at $x=0, u(0, t)$ and $u_{x}(0, t)$ exhibit single exponential oscillations:

$$
u(0, t)=\tilde{u}_{1}(t)(1+o(1)) \quad \text { and } \quad u_{x}(0, t)=\tilde{u}_{2}(t)(1+o(1)), \quad t \rightarrow \infty,
$$

where

$$
\begin{gathered}
\tilde{u}_{1}(t)=\mathrm{e}^{-\mathrm{i}\left(\eta-\frac{\pi}{2}\right)} \frac{\sqrt{2 \omega}}{\cosh \left(\phi_{0}\right)} \mathrm{e}^{2 \mathrm{i} \omega t}, \\
\tilde{u}_{2}(t)=-\mathrm{e}^{-\mathrm{i}\left(\eta-\frac{\pi}{2}\right)} \frac{2 \omega \sinh \left(\phi_{0}\right)}{\cosh ^{2}\left(\phi_{0}\right)} \mathrm{e}^{2 \mathrm{i} \omega t} .
\end{gathered}
$$

Remark 5.1. For $\beta<0$, the asymptotic analysis for the domain $\frac{x}{t}>4|\beta|$ is similar to that in the case of step-like initial data considered in [5], giving the asymptotics in the form of a modulated elliptic wave for $4|\beta|<\frac{x}{t}<4|\beta|+4 \sqrt{2} \alpha$ and a plane wave for $\frac{x}{t}>4|\beta|+4 \sqrt{2} \alpha$.

\section{Acknowledgments}

D.Sh. gratefully acknowledges the support of the Austrian Science Fund (FWF) under Grant No. Y330 and the hospitality of the Archimedes Center for Modeling, Analysis and Computation (University of Crete), Laboratoire de Mathématiques Pures et Appliquées (Université du Littoral Côte d'Opale), and the University of Vienna, where parts of this research were done.

S.K. acknowledges the support of the European Unions Seventh Framework Programme (FP7REGPOT-2009-1) under grant agreement n 245749, as well as the ARISTEIA II grant n. 3964 from the Greek General Secretariat of Research and Technology.

\section{References}

[1] R.F. Bikbaev and V.O. Tarasov, Initial-boundary value problem for the nonlinear Schrödinger equation, J. Phys. A: Math. Gen. 24, no.11 (1991) 2507-2516.

[2] R.F. Bikbaev and A.R. Its, Algebrogeometric solutions of the boundary problem for the nonlinear Schrödinger equation, Mathematical Notes 45, no. 5 (1989) 349-354 (Russian original: Mat. Zam. 45, no.5 (1989) 3-9).

[3] A. Boutet de Monvel, A.S. Fokas, and D. Shepelsky, Analysis of the global relation for the nonlinear Schrödinger equation on the half-line, Lett. Math. Phys. 65 (2003) 199-212.

[4] A. Boutet de Monvel, A.S. Fokas, and D. Shepelsky, The mKdV equation on the half-line, J. Inst. Math. Jussieu 3 (2004) 139-164.

[5] A. Boutet de Monvel, V.P. Kotlyarov, and D. Shepelsky, Focusing NLS equation: long-time dynamics of step-like initial data, Internat. Math. Res. Notices 7 (2011) 1613-1653.

[6] R. Buckingham and S. Venakides, Long-time asymptotics of the nonlinear Schrödinger equation shock problem, Communications on Pure and Applied Mathematics 60, no. 9 (2007) 1349-1414.

[7] P. Deift and J. Park, Long-time asymptotics for solutions of the NLS equation with a delta potential and even initial data, Int. Math. Res. Notices (2011) doi:10.1093/imrn/rnq282.

[8] P. Deift and X. Zhou, A steepest descent method for oscillatory Riemann-Hilbert problems. Asymptotics for the MKdV equation, Ann. of Math. 137, no. 2 (1993) 295-368.

[9] L.D. Faddeev and L.A. Takhtajan, Hamiltonian methods in the theory of solitons. Translated from the Russian by A. G. Reyman. (Springer Series in Soviet Mathematics. Springer-Verlag, Berlin, 1987).

[10] A.S. Fokas, An initial-boundary value problem for the nonlinear Schrödinger equation, Physica D 35, no.1-2 (1989), 167-185.

[11] A.S. Fokas, Initial boundary-value problems for soliton equations, in: Nonlinear Processes in Physics, eds. A.S. Fokas, D.J. Kaup, A.C. Newell, V.E. Zakharov ( Springer-Verlag, 1993). 
[12] A.S. Fokas, Integrable nonlinear evolution equations on the half-line, Comm. Math. Phys. 230 (2002) 1-39.

[13] A.S. Fokas and A.R. Its, An initial-boundary value problem for the Korteweg-de Vries equation. Solitons, nonlinear wave equations and computation (New Brunswick, NJ, 1992). Math. Comput. Simulation 37, no. 4-5 (1994) 293-321.

[14] A.S. Fokas and A.R. Its, The linearization of the initial boundary value problem of the nonlinear Schrödinger equation, SIAM J. Math. Anal. 27, no.3 (1996) 738-764.

[15] A.S. Fokas and S. Kamvissis, Zero dispersion limit for integrable equations on the half-line with linearizable data, Abstract and Applied Analysis 5 (2004) 361-370.

[16] A.S. Fokas, J. Lenells, Explicit soliton asymptotics for the Korteweg-de Vries equation on the half-line, Nonlinearity 23, no. 4 (2010) 937-976.

[17] A.S. Fokas, A.R. Its, and L-Y. Sung, The nonlinear Schrödinger equation on the half-line, Nonlinearity 18, no.4 (2005) 1771-1822.

[18] J. Holmer and M. Zvorski, Breathing pattern in nonlinear relaxation, Nonlinearity 22, no.6 (2009) 1259-1301.

[19] A. Its and D. Shepelsky, Initial boundary value problem for the focusing NLS equation with Robin boundary condition: half-line approach, Proc. R. Soc. A. 469 (2013) 20120199.

[20] E.K. Sklyanin, Boundary conditions for integrable equations, Funct. Anal. Appl. 21 (1987) 86-87.

[21] V.O. Tarasov, A boundary value problem for the nonlinear Schrödinger equation, J. Soviet Math. 54, no. 3 (1991) 958-967 (Russian original: Zap. Nauchn. Sem. LOMI 169(3) (1988) 151-165).

[22] V.O. Tarasov, The integrable initial-boundary value problem on a semi-line: nonlinear Schrödinger and sine-Gordon equations, Inverse Problems 7, no.3 (1991) 435-449.

[23] S. Venakides, P. Deift, and R. Oba, The Toda shock problem Comm. Pure Appl. Math. 44, no. 8-9 (1991) 1171-1242.

[24] V.E. Zakharov and A.B. Shabat, Exact Theory of Two-dimensional Self-focusing and One-dimensional Self-modulation of Waves in Nonlinear Media, Sov. Phys. JETP 34(1) (1972) 62-69 (Russian original: Zh.Eksp. Teor. Fiz. 61(1) (1971) 118-134). 